\title{
INTEGRAL DEFERRED CORRECTION METHODS CONSTRUCTED WITH HIGH ORDER RUNGE-KUTTA INTEGRATORS
}

\author{
ANDREW CHRISTLIEB, BENJAMIN ONG, AND JING-MEI QIU
}

\begin{abstract}
Spectral deferred correction (SDC) methods for solving ordinary differential equations (ODEs) were introduced by Dutt, Greengard and Rokhlin (2000). It was shown in that paper that SDC methods can achieve arbitrary high order accuracy and possess nice stability properties. Their SDC methods are constructed with low order integrators, such as forward Euler or backward Euler, and are able to handle stiff and non-stiff terms in the ODEs. In this paper, we use high order Runge-Kutta (RK) integrators to construct a family of related methods, which we refer to as integral deferred correction (IDC) methods. The distribution of quadrature nodes is assumed to be uniform, and the corresponding local error analysis is given. The smoothness of the error vector associated with an IDC method, measured by the discrete Sobolev norm, is a crucial tool in our analysis. The expected order of accuracy is demonstrated through several numerical examples. Superior numerical stability and accuracy regions are observed when high order RK integrators are used to construct IDC methods.
\end{abstract}

\section{INTRODUCTION}

In this paper, we consider integral deferred correction methods (IDC) 44 for solving initial value problems (IVP) consisting of ordinary differential equations (ODEs). Note that in the literature, this family of methods is referred to as spectral deferred correction (SDC) methods if Gaussian quadrature nodes are used to compute a correction to the defect or error. Compared to traditional multistep methods, e.g., Adams-Bashforth methods (AB) and multi-stage methods, e.g., Runge-Kutta (RK) methods, IDC methods are able to achieve arbitrary high order accuracy without tedious algebraic computations and are able to handle stiff and non-stiff terms in the ODEs. IDC methods also maintain reasonably large stability regions as the order of the schemes increases.

As discussed in [4, there are various decisions to be made when constructing IDC methods; much work has been done in the literature to explore these choices. The selection of quadrature nodes is discussed in [11, while [15] uses semi-implicit schemes to handle temporal multi-scale problems. The authors in [13, 12] also

Received by the editor April 3, 2008 and, in revised form, October 12, 2008 and March 21, 2008.

2000 Mathematics Subject Classification. Primary 65B05.

Key words and phrases. Integral deferred correction methods, Runge-Kutta methods, discrete Sobolev norm, local error, accuracy, stability.

Research supported by Air Force Office of Scientific Research and Air Force Research Labs (Edwards and Kirtland). Grant Numbers FA9550-07-1-0092 and FA9550-07-1-0144. 
study the choice of predictors and correctors to construct semi-implicit SDC methods. In [8, 9, Krylov subspace methods are used to accelerate the convergence of SDC methods. In [7, 6], the smoothness of the error vector associated with an SDC method is introduced and is used to discuss the convergence of SDC schemes. Their analysis and concept of smoothness of the error vector motivated this paper. Theoretical convergence results for SDC methods constructed using low order integrators are discussed in various papers [5, 1, 18. The application of SDC methods to PDEs, through the method of lines approach, can be found in [16, 10, 2, 18, 14.

The main focus of this paper is to study IDC methods constructed using high order RK integrators. Specifically, we will prove, under mild conditions, that using an $r^{t h}$ order RK integrator to solve the error equation in a correction loop increases the order of accuracy of an IDC method by $r$ orders. The smoothness of the error vector associated with an IDC method, measured by a discrete Sobolev norm (introduced and used in [7, 17]), is a crucial concept in the local error analysis. In contrast to the SDC methods in [4, we assume that the quadrature nodes are uniformly distributed. For the case of a non-uniform distribution of quadrature nodes, including the Gaussian quadrature nodes discussed in 4, preliminary numerical results indicate that a corresponding relation for the order increase does not hold. We address these issues in 3 .

The main part of the paper is organized into six sections. \2 is a review of IDC methods described in [4, while $\$ 3$ introduces the concept of discrete smoothness, measured by a discrete Sobolev norm. $₫$ gives an analysis of the local error of IDC methods constructed with forward Euler integrators; this section sets a framework for the analysis of IDC methods constructed using high order RK methods in $\$ 5$. In 96 a numerical example is provided to support the theoretical results in $\$ 4$ and 95. Superior stability and accuracy properties of IDC methods constructed with high order RK integrators are also demonstrated. Concluding remarks are given in $\S 7$

\section{REVIEW OF IDC METHODS}

This section is a review of IDC methods from [4]. Our discussion of these methods is based on the notation introduced below. We consider an IVP consisting of a system of ODEs and initial conditions,

$$
\left\{\begin{array}{l}
y^{\prime}(t)=f(t, y), \quad t \in[0, T] \\
y(0)=y_{0}
\end{array}\right.
$$

The time domain, $[0, T]$, is discretized into intervals,

$$
0=t_{1}<t_{2}<\cdots<t_{n}<\cdots<t_{N}=T,
$$

and each interval, $I_{n}=\left[t_{n}, t_{n+1}\right]$, is further discretized into subintervals,

$$
t_{n}=t_{n, 0}=t_{n, 1}<\cdots<t_{n, m}<\cdots<t_{n, M}=t_{n+1} .
$$

The IDC method on each time interval $\left[t_{n}, t_{n+1}\right]$ is described below. We drop the subscript $n$, e.g., $t_{0}:=t_{n, 0}$ in (2.2), with the understanding that the IDC method is described for one time interval. We also refer to $t_{m}:=t_{n, m}$ as grid points or quadrature nodes, whose index $m$ runs from 0 to $M$, and we denote the subinterval sizes as $h_{m}=t_{m}-t_{m-1}, m=1, \ldots, M$.

- (prediction step) Use an $\left(r_{0}\right)^{t h}$ order numerical method to obtain a numerical solution, $\vec{\eta}^{[0]}=\left(\eta_{0}^{[0]}, \eta_{1}^{[0]}, \ldots, \eta_{m}^{[0]}, \ldots, \eta_{M}^{[0]}\right)$, which is an $r_{0}^{\text {th }}$ order 
approximation to $\vec{y}=\left(y_{0}, y_{1}, \ldots, y_{m}, \ldots, y_{M}\right)$, where $y_{m}=y\left(t_{m}\right)$ is the exact solution at $t_{m}$. For example, applying a first order forward Euler method to (2.1) gives $\eta_{m+1}^{[0]}=\eta_{m}^{[0]}+h_{m+1} f\left(t, \eta_{m}^{[0]}\right), m=0, \ldots, M-1$.

- (correction loop) Use the error function to improve the accuracy of the scheme at each iteration.

For $k=1, \ldots, k_{l}$ ( $k_{l}$ is the number of correction steps)

(1) Denote the error function from the previous step as

$$
e^{(k-1)}(t)=y(t)-\eta^{(k-1)}(t),
$$

where $y(t)$ is the exact solution and $\eta^{(k-1)}(t)$ is an $M^{t h}$ degree polynomial interpolating $\vec{\eta}^{[k-1]}$. Note that the error function, $e^{(k-1)}(t)$, is not a polynomial in general.

(2) Compute the residual function, $\epsilon^{(k-1)}(t)=\left(\eta^{(k-1)}\right)^{\prime}(t)-f\left(t, \eta^{(k-1)}(t)\right)$. In the literature, the residual function is often called the pointwise defect.

(3) Compute the numerical error vector, $\vec{\delta}^{[k]}=\left(\delta_{0}^{[k]}, \ldots, \delta_{m}^{[k]}, \ldots, \delta_{M}^{[k]}\right)$, using an $\left(r_{k}\right)^{\text {th }}$ order numerical method to discretize the integral form of the error equation,

$$
\begin{aligned}
\left(e^{(k-1)}+\int_{0}^{t} \epsilon^{(k-1)}(\tau) d \tau\right)^{\prime}(t) & =f\left(t, \eta^{(k-1)}(t)+e^{(k-1)}(t)\right)-f\left(t, \eta^{(k-1)}(t)\right) \\
& \doteq F\left(t, e^{(k-1)}(t)\right)
\end{aligned}
$$

where $F(t, e(t))=f(t, \eta(t)+e(t))-f(t, \eta(t)) . \vec{\delta}^{[k]}$ is an $\left(r_{k}\right)^{t h}$ order approximation to $\vec{e}^{[k-1]}=\left(e_{0}^{[k-1]}, \ldots, e_{m}^{[k-1]}, \ldots, e_{M}^{[k-1]}\right)$, and $e_{m}^{[k-1]}=$ $e^{(k-1)}\left(t_{m}\right)$ is the value of the exact error function at $t_{m}$. For example, applying a first order forward Euler method to (2.4) gives

$$
\begin{array}{r}
\delta_{m+1}^{[k]}=\delta_{m}^{[k]}+h_{m+1}\left(f\left(t_{m}, \eta_{m}^{[k-1]}+\delta_{m}^{[k]}\right)-f\left(t_{m}, \eta_{m}^{[k-1]}\right)\right)-\int_{t_{m}}^{t_{m+1}} \epsilon^{(k-1)}(t) d t \\
\approx \delta_{m}^{[k]}+h_{m+1}\left(f\left(t_{m}, \eta_{m}^{[k-1]}+\delta_{m}^{[k]}\right)-f\left(t_{m}, \eta_{m}^{[k-1]}\right)\right)-\sum_{j=0}^{M} \alpha_{m, j} f\left(t_{j}, \eta_{j}^{[k-1]}\right) \\
m=0, \ldots, M-1
\end{array}
$$

where we have approximated the integral by interpolatory quadrature formulas, as in [4.

(4) Update the numerical solution $\vec{\eta}^{[k]}=\vec{\eta}^{[k-1]}+\vec{\delta}^{[k]}$.

Notationally, superscripts with a round bracket, e.g., $(k)$, denote a function, while superscripts with a square bracket, e.g., $[k]$, denote a vector at the $k^{\text {th }}$ correction step. English letters are reserved for functions or vectors in the exact solution space, e.g., an exact solution $y(t)$ and an exact error function $e(t)$, while Greek letters denote functions or vectors in the numerical solution space, e.g., a numerical solution $\eta(t)$, and a numerical error function $\delta(t)$.

Remark 2.1. There are various construction decisions for generating an IDC method, for example, the distribution of grid points (2.2), or the choice of different integrators. In this paper, we would like to make the statements " $\left(r_{0}\right)^{\text {th }}$ order approximation" and " $\left(r_{k}\right)^{\text {th }}$ order approximation" in our description of IDC methods 
mathematically rigorous. We will focus our discussion on a uniform distribution of quadrature nodes. The non-uniform case, including Gaussian quadrature nodes, will be addressed in $[3$.

Remark 2.2. Deferred correction methods described in reference [4] and cited therein differ from the spectral deferred correction in that a differential form of the error equation is formulated,

$$
\left(e^{(k-1)}\right)^{\prime}(t)=f\left(t, \eta^{(k-1)}(t)+e^{(k-1)}(t)\right)-\frac{\partial}{\partial t} \eta^{(k-1)}(t),
$$

instead of (2.4). In this paper, we focus on IDC methods and briefly address the corresponding results in deferred correction methods.

\section{Mathematical PREliminaries}

Definition 3.1 (smoothness of a function). A function $f(t), t \in[0, T]$, possesses $S$ degrees of smoothness if $\left\|d^{s} f\right\|_{\infty}:=\left\|\frac{\partial^{s}}{\partial t^{s}} f\right\|_{\infty}$ is bounded, for $s=0,1,2, \ldots, S$, where $\|f\|_{\infty} \doteq \max _{t \in[0, T]}|f(t)|$.

We would like to establish the concept of smoothness for a discrete data set, analogous to that of a function. Consider the discrete data set,

$$
(t, f)=\left\{\left(t_{0}, f_{0}\right), \ldots,\left(t_{M}, f_{M}\right)\right\},
$$

where $f_{m}=f\left(t_{m}\right)$ and $t_{m}=m h, m=0, \ldots, M$ are equispaced points. The smoothness of a discrete data set is established in the limiting process of $h \rightarrow 0$.

Definition 3.2 (discrete differentiation). Given a discrete data set, $(t, f)$, defined in (3.1), denote $L^{M}$ as the usual Lagrange interpolant, an $M^{\text {th }}$ degree polynomial that interpolates $(t, f)$,

$$
L^{M}(t, f)=\sum_{m=0}^{M} c_{m}(t) f_{m}, \quad \text { where } \quad c_{m}(t)=\prod_{n \neq m} \frac{t-t_{n}}{t_{m}-t_{n}} .
$$

An $s^{t h}$ degree discrete differentiation is a linear mapping that maps $\vec{f}=\left(f_{0}, f_{1}, \ldots\right.$, $\left.f_{M}\right)$ into $\overrightarrow{\hat{d}_{s} f}$, where $\left(\hat{d}_{s} f\right)_{m}=\left.\frac{\partial^{s}}{\partial t^{s}} L^{M}(t, f)\right|_{t=t_{m}}$. This linear mapping can be represented by a matrix multiplication $\overrightarrow{\hat{d}_{s} f}=D_{s} \cdot \vec{f}$, where $D_{s} \in \mathcal{R}^{(M+1) \times(M+1)}$, and $\left(D_{s}\right)_{m n}=\left.\frac{\partial^{s}}{\partial t^{s}} c_{n}(t)\right|_{t=t_{m}}, m, n=0, \ldots, M$.

Definition 3.3. The $(\hat{S}, \infty)$ Sobolev norm of the discrete data set $(t, f)$ is defined to be

$$
\|\vec{f}\|_{\hat{S}, \infty}=\sum_{s=0}^{S}\left\|\overrightarrow{\hat{d}_{s} f}\right\|_{\infty}=\sum_{s=0}^{S}\left\|D_{s} \cdot \vec{f}\right\|_{\infty},
$$

where $\overrightarrow{\hat{d}_{0} f}=I d \cdot \vec{f}$ is the identity matrix operating on $\vec{f}$.

Definition 3.4 (smoothness of a discrete data set). A discrete data set (3.1), possesses $S(S \leq M)$ degrees of smoothness if $\|\vec{f}\|_{\hat{S}, \infty}$ is bounded as $h \rightarrow 0$.

The smoothness of a discrete data set can also be measured by divided difference approximations to the derivative of the discrete data set. 
Definition 3.5 (divided differences). Given a discrete data set $(t, f)$ as in (3.1), a divided difference of the discrete data set is a linear mapping that maps $\vec{f}=$ $\left(f_{0}, f_{1}, \ldots, f_{M}\right)$ into $\overrightarrow{d_{1} f}$, with size $\left(\overrightarrow{d_{1} f}\right)=M$ and $\left(d_{1} f\right)_{m}=\frac{f_{m+1}-f_{m}}{t_{m+1}-t_{m}}$ for $m=$ $0, \ldots, M-1$. An $s^{\text {th }}$ degree divided difference is a linear mapping that maps $\vec{f}$ into $\overrightarrow{d_{s} f}$, with size $\left(\overrightarrow{d_{s} f}\right)=M-s+1$ and $\left(d_{s} f\right)_{m}=\frac{\left(d_{s-1} f\right)_{m+1}-\left(d_{s-1} f\right)_{m}}{t_{m+1}-t_{m}}$, for $m=$ $0, \ldots, M-s$.

Definition 3.6. The $(S, \infty)$ Sobolev norm of $(t, f)$ is defined to be

$$
\|\vec{f}\|_{S, \infty}=\sum_{s=0}^{S}\left\|\overrightarrow{d_{s} f}\right\|_{\infty},
$$

where $\overrightarrow{d_{0} f}=I d \cdot f$.

Proposition 3.7. The $(\hat{S}, \infty)$ Sobolev norm defined in Definition 3.3 is equivalent to the $(S, \infty)$ Sobolev norm defined in Definition 3.6.

Proof. We will prove the equivalence of the $(\hat{1}, \infty)$ and $(1, \infty)$ norms; the equivalence of the general $(\hat{S}, \infty)$ and $(S, \infty)$ norms can be proved in a similar fashion. For any discrete data set (3.1), let $L^{M}(t, f)$ be the corresponding Lagrange interpolant (3.2). By Definition 3.2. $\left(\hat{d}_{1} f\right)_{m}=\left.\frac{\partial}{\partial t} L^{M}(t, f)\right|_{t=t_{m}} \cdot\left(d_{1} f\right)_{m}$ is a first order approximation to $\left.\frac{\partial}{\partial t} L^{M}(t, f)\right|_{t=t_{m}}$, i.e.,

$$
\left(d_{1} f\right)_{m}=\left.\frac{\partial}{\partial t} L^{M}(t, f)\right|_{t=t_{m}}+\mathcal{O}(h)=\left(\hat{d}_{1} f\right)_{m}+\mathcal{O}(h) .
$$

From (3.3), if the $(\hat{1}, \infty)$ norm is bounded and independent of $H$, then so is the $(1, \infty)$ norm, and vice versa. This proves the equivalence of the two norms.

Utilizing Proposition [3.7 the smoothness of a discrete data set can also be measured by divided differences of the discrete data set.

Definition 3.8. (smoothness of a discrete data set) A discrete data set (3.1), possesses $S(S \leq M)$ degrees of smoothness if $\|\vec{f}\|_{S, \infty}$ is bounded, as $h \rightarrow 0$.

Remark 3.9. We require $S \leq M$ in Definition 3.4 and Definition 3.8 because $\overrightarrow{\hat{d}_{M+1} f}=\overrightarrow{0}$ and $\operatorname{size}\left(\overrightarrow{d_{M+1} f}\right)=0$, for any discrete data set.

Example 3.10 (a not so smooth discrete data set). The discrete data set,

$$
(t, f)=\{(0,0),(h, h),(2 h, 2 h),(3 h, h),(4 h, 0)\},
$$

with $H=4 h$ has only one degree of smoothness in the discrete sense, according to either Definition 3.4 or Definition 3.8 .

We will use the definitions of smoothness, Definition 3.4 and Definition 3.8 interchangeably as convenient. Propositions 3.11 through 3.15 describe basic properties associated with the smoothness of discrete data sets. We omit the proofs for brevity.

Proposition 3.11. If a discrete data set $(t, f)$, as defined in (3.1), has $S$ degrees of smoothness, then $\left(t, h^{p} f\right)=\left\{\left(t_{m}, h^{p} \cdot f_{m}\right)\right\}_{m=0}^{M}$ has $\min (S+p, M)$ degrees of smoothness.

Proposition 3.12. If discrete data sets $(t, f)$ and $(t, g)$ have $S_{f}$ and $S_{g}$ degrees of smoothness, respectively, then $(t, f+g)=\left\{\left(t_{m}, f_{m}+g_{m}\right)\right\}_{m=0}^{M}$ and $(t, f \cdot g)=$ $\left\{\left(t_{m}, f_{m} \cdot g_{m}\right)\right\}_{m=0}^{M}$ have $\min \left(S_{f}, S_{g}\right)$ degrees of smoothness. 
Proposition 3.13. If a function $f(t), t \in[0, H]$, has $S$ degrees of smoothness in the continuous sense, then the discrete data set $(t, f)=\left\{\left(t_{m}, f\left(t_{m}\right)\right)\right\}_{m=0}^{M}$ has $\min (S, M)$ degrees of smoothness in the discrete sense.

Proposition 3.14. If a function $f(t), t \in[0, H]$, has $S$ degrees of smoothness in the continuous sense, then the discrete data set $\left(t, H^{p} \cdot \frac{d^{q} f}{d t^{q}}\right)=\left\{\left(t_{m}, H^{p} \frac{d^{q} f}{d t^{q}}\left(t_{m}\right)\right)\right\}_{m=0}^{M}$ has $\min (S-q+p, M)$ degrees of smoothness in the discrete sense with $q \leq S$. This is a direct consequence from Proposition 3.13 and Proposition 3.11 .

Proposition 3.15. If a function $f(t)$ has $S_{f}$ degrees of smoothness in the continuous sense and the discrete data set $(t, g)=\left\{t_{m}, g_{m}\right\}_{m=0}^{M}$ has $S_{g}$ degrees of smoothness in the discrete sense, then the discrete data set $(t, f \circ g)=\left\{t_{m}, f\left(g_{m}\right)\right\}_{m=0}^{M}$ has $\min \left(S_{f}, S_{g}\right)$ degrees of smoothness in the discrete sense.

Finally, we establish the relationship between the smoothness of the error vector associated with an IDC method, measured by properties of its discrete derivative or its divided difference, and the derivative of the corresponding error function.

Proposition 3.16. Let $y(t)$ in IVP (2.1) have $S \geq M+2$ degrees of smoothness in the continuous sense, and let $\eta$ be the numerical solution computed using an IDC method constructed with $(M+1)$ equispaced quadrature nodes in each subinterval. If the error vector associated with an IDC method, $\vec{e}=\vec{y}-\vec{\eta}$, satisfies $\|\vec{e}\|_{\infty} \sim \mathcal{O}\left(h^{r+1}\right)$ with $r \leq M$, and the corresponding error function from interpolation errors, $e(t)=$ $y(t)-L^{M}(t, \eta)$, satisfies $e_{i}=e\left(t=t_{i}\right)$ (note that $e(t)$ is not a polynomial in general), then

- $e(t) \sim \mathcal{O}\left(h^{r+1}\right)$, for $t \in[0, H]$.

- Let $\overrightarrow{\tilde{e}}=\frac{1}{h^{r}} \vec{e}$ and $\tilde{e}(t)=\frac{1}{h^{r}} e(t)$. If $\overrightarrow{\tilde{e}}$ has $S_{\tilde{e}} \leq M+1-r$ degrees of smoothness in the discrete sense, then

$$
\frac{d^{i}}{d t^{i}} \tilde{e}(t) \sim\left\{\begin{array}{ll}
\mathcal{O}(1), & \text { if } 0 \leq i \leq S_{\tilde{e}} \\
\mathcal{O}\left(\frac{1}{h^{i-S_{\tilde{e}}}}\right), & \text { if } S_{\tilde{e}} \leq i \leq M+1-r,
\end{array} \quad \forall t \in[0, H] .\right.
$$

- The vector $\left(\left.\frac{d^{i} \tilde{e}(t)}{d t^{i}}\right|_{t=t_{0}}, \ldots,\left.\frac{d^{i} \tilde{e}(t)}{d t^{i}}\right|_{t=t_{M}}\right)$ has $S_{\tilde{e}}-i$ degrees of smoothness in the discrete sense for $i \leq S_{\tilde{e}}$.

The proof of this proposition can be inferred from well-known properties of the derivatives in Lagrange interpolation. Note that these error estimates for polynomial interpolation do not require that the grid be equispaced.

\section{LOCAL ERROR OF IDC METHODS CONSTRUCTED WITH FORWARD EULER INTEGRATORS}

In this section, we provide local error estimates for IDC methods constructed using forward Euler integrators. Our approach differs from the convergence and local error estimates discussed in [8, 18, respectively. This section introduces the framework which allows for the analysis of IDC methods constructed using high order RK methods, discussed in Section 5. In the following theorems and lemmas, we will assume that function $f(t, y)$ in IVP (2.1) has $S-1$ degrees of smoothness if the solution $y(t)$ has $S$ degrees of smoothness.

Theorem 4.1. Let $y(t)$, the solution to the IVP (2.1), have at least $S \geq M+2$ degrees of smoothness in the continuous sense. Then, the local error for an IDC method constructed using $(M+1)$ uniformly distributed nodes $\left(t_{m}=m h, m=\right.$ 
$0, \ldots, M)$, and forward Euler integrators for the prediction and $k_{l} \leq M$ correction loops, is $\mathcal{O}\left(h^{k_{l}+2}\right)$.

Proof. The proof of Theorem 4.1 follows from two lemmas discussed in depth below. We will show in Lemma 4.2 that the theorem holds for $k=0$, and in Lemma 4.3 that an inductive argument is satisfied. Note that this theorem is also implied by discussions in [5].

Lemma 4.2 (prediction step). Consider an IDC method constructed using $(M+1)$ uniformly distributed nodes and a forward Euler integrator for the prediction step. Let $y(t)$, the solution to the IVP (2.1), have at least $S \geq M+2$ degrees of smoothness, and let $\vec{\eta}^{[0]}=\left(\eta_{0}^{[0]}, \ldots, \eta_{m}^{[0]}, \ldots, \eta_{M}^{[0]}\right)$ be the numerical solution computed after the prediction step. Then the error vector, $\vec{e}^{[0]}=\vec{y}-\vec{\eta}^{[0]}$, satisfies $\left\|\vec{e}^{[0]}\right\|_{\infty} \sim \mathcal{O}\left(h^{2}\right)$, and the rescaled error vector, $\overrightarrow{\tilde{e}}^{[0]}=\frac{1}{h} \vec{e}^{[0]}$, has $M$ degrees of smoothness in the discrete sense.

Proof. We drop the superscript [0] as there is no ambiguity. Since $\eta_{m+1}=\eta_{m}+$ $h f\left(t_{m}, \eta_{m}\right)$, the error at $t_{m+1}, e_{m+1}=y_{m+1}-\eta_{m+1}$, satisfies

$$
e_{m+1}=e_{m}+h\left(f\left(t_{m}, y_{m}\right)-f\left(t_{m}, \eta_{m}\right)\right)+\sum_{i=2}^{S-1} \frac{h^{i}}{i !} y^{(i)}\left(t_{m}\right)+\mathcal{O}\left(h^{S}\right),
$$

where we have performed a Taylor expansion of $y_{m+1}$ about $t=t_{m}$. Let $u_{m}=$ $f\left(t_{m}, y_{m}\right)-f\left(t_{m}, \eta_{m}\right)$ and let $r_{m}=\frac{h^{2}}{2 !} y^{(2)}\left(t_{m}\right)+\cdots+\frac{h^{S-1}}{(S-1) !} y^{(S-1)}\left(t_{m}\right)$. Notice that

$$
u_{m}=e_{m} f_{y}\left(t_{m}, y_{m}\right)+\cdots+\frac{(-1)^{S-1}\left(e_{m}\right)^{S-2}}{(S-2) !} f_{y^{S-2}}\left(t_{m}, y_{m}\right)+\mathcal{O}\left(\left(e_{m}\right)^{S-1}\right),
$$

where we have performed a Taylor expansion of $f\left(t, \eta_{m}\right)$ about $y=y_{m}$. We are now ready to bound $\left\|\vec{e}^{[0]}\right\|_{\infty}$ by induction. By definition, $e_{0}=0$, so certainly, $e_{0} \sim \mathcal{O}\left(h^{2}\right)$. Assume that $e_{m} \sim \mathcal{O}\left(h^{2}\right)$. Since $u_{m} \sim \mathcal{O}\left(e_{m}\right) \sim \mathcal{O}\left(h^{2}\right)$, we have

$$
e_{m+1}=e_{m}+h u_{m}+r_{m}+\mathcal{O}\left(h^{S}\right) \sim \mathcal{O}\left(h^{2}\right),
$$

which completes the inductive proof that $\|\vec{e}\|_{\infty} \sim \mathcal{O}\left(h^{2}\right)$. Note that the inductive proof was with respect to $m$, the index of the grid points.

To prove the smoothness of the rescaled error vector, we will again use an inductive approach, but this time, with respect to $s$, the degree of smoothness. First, note that a divided difference approximation to the derivative of the rescaled error vector gives

$$
\left(d_{1} \tilde{e}\right)_{m}=\frac{\tilde{e}_{m+1}-\tilde{e}_{m}}{h}=\tilde{u}_{m}+\frac{r_{m}}{h^{2}}+\mathcal{O}\left(h^{S-2}\right),
$$

where

$$
\tilde{u}_{m}=\frac{u_{m}}{h}=\sum_{i=1}^{S-2}(-1)^{i+1} \frac{h^{i-1}}{i !} f_{y^{i}}\left(t_{m}, y_{m}\right)\left(\tilde{e}_{m}\right)^{i}+\mathcal{O}\left(h^{2 S-3}\right) .
$$

We are now ready to prove that $\overrightarrow{\tilde{e}}$ has $M$ degrees of smoothness by induction. Since $\|\overrightarrow{\tilde{e}}\|_{\infty} \sim \mathcal{O}(h), \overrightarrow{\tilde{e}}$ has at least zero degrees of smoothness in the discrete sense. Assume that $\overrightarrow{\tilde{e}}$ has $s \leq M-1$ degrees of smoothness. We will show that $\overrightarrow{d_{1}} \overrightarrow{\tilde{e}}$ has $s$ degrees of smoothness, from which we can conclude that $\overrightarrow{\tilde{e}}$ has $(s+1)$ degrees of smoothness. 
Since $f_{y^{i}}$ has $(S-i-1)$ degrees of smoothness in the continuous sense, $\overrightarrow{f_{y^{i}}}=$ $\left[f_{y^{i}}\left(t_{0}, y_{0}\right), \ldots, f_{y^{i}}\left(t_{M}, y_{M}\right)\right]$ has $(S-i-1)$ degrees of smoothness in the discrete sense. Consequently, $h^{i-1} \overrightarrow{f_{y^{i}}}$ has $(S-2)$ degrees of smoothness, which implies that $\overrightarrow{\tilde{u}}$ has $\min (S-2, s)$ degrees of smoothness. Similarly, $\frac{\vec{r}}{h^{2}}$ has $(S-2)$ degrees of smoothness in the discrete sense. Hence $\overrightarrow{d_{1} \tilde{e}}$ has $s$ degrees of smoothness $\Longrightarrow \overrightarrow{\tilde{e}}$ has $(s+1)$ degrees of smoothness. Since this argument holds for $S \geq M+2$, we can conclude that $\overrightarrow{\tilde{e}}$ has $M$ degrees of smoothness.

Lemma 4.3 (correction step). Consider an IDC method constructed using $(M+1)$ uniformly distributed nodes and forward Euler integrators for the prediction and $k_{l} \leq M$ correction loops. Also, let $y(t)$, the solution to the IVP (2.1), have at least $S \geq M+2$ degrees of smoothness, and let $\vec{\eta}^{[k]}=\left(\eta_{0}^{[k]}, \ldots, \eta_{m}^{[k]}, \ldots, \eta_{M}^{[k]}\right)$ be the numerical solution computed after the $k^{\text {th }}$ correction loop. If $\vec{e}^{[k-1]} \sim \mathcal{O}\left(h^{k+1}\right)$ and $\overrightarrow{\tilde{e}}^{[k-1]}=\frac{1}{h^{k}} \vec{e}^{[k-1]}$ has $(M-k+1)$ degrees of smoothness, then $\left\|\vec{e}^{[k]}\right\|_{\infty} \sim \mathcal{O}\left(h^{k+2}\right)$, and $\overrightarrow{\tilde{e}}^{[k]}=\frac{1}{h^{k+1}} \vec{e}^{[k]}$ has $(M-k)$ degrees of smoothness in the discrete sense.

Proof. The error equation, (2.4), integrated over $\left[t_{m}, t_{m+1}\right]$, gives

$$
\begin{aligned}
e_{m+1}^{[k-1]}= & e_{m}^{[k-1]}+\int_{t_{m}}^{t_{m+1}} F\left(t, e^{(k-1)}(t)\right) d t-\int_{t_{m}}^{t_{m+1}} \epsilon^{(k-1)}(t) d t \\
= & e_{m}^{[k-1]}+h F\left(t_{m}, e_{m}^{[k-1]}\right)+\sum_{i=1}^{M} \frac{h^{i+1}}{(i+1) !} \frac{d^{i}}{d t^{i}} F\left(t_{m}, e_{m}^{[k-1]}\right) \\
& +\mathcal{O}\left(h^{M+2}\right)-\int_{t_{m}}^{t_{m+1}} \epsilon^{(k-1)}(t) d t
\end{aligned}
$$

Recall from equation (2.5) that the numerical error vector, which arises from utilizing a forward Euler integrator to solve the error equation, satisfies

$$
\begin{aligned}
\delta_{m+1}^{[k]}=\delta_{m}^{[k]}+h\left(f \left(t_{m},\right.\right. & \left.\left.\eta_{m}^{[k-1]}+\delta_{m}^{[k]}\right)-f\left(t_{m}, \eta_{m}^{[k-1]}\right)\right) \\
& -\left(\int_{t_{m}}^{t_{m+1}} \epsilon^{(k-1)}(t) d t+\mathcal{O}\left(h^{M+2}\right)\right) .
\end{aligned}
$$

Subtracting the numerical error vector from the error equation gives

$$
\begin{aligned}
e_{m+1}^{[k]}=e_{m}^{[k]} & +h\left(f\left(t_{m}, y_{m}\right)-f\left(t_{m}, \eta_{m}^{[k-1]}+\delta_{m}^{[k]}\right)\right) \\
& +\sum_{i=1}^{M} \frac{h^{i+1}}{(i+1) !} \frac{d^{i}}{d t^{i}} F\left(t_{m}, e_{m}^{[k-1]}\right)+\mathcal{O}\left(h^{M+2}\right) \\
= & e_{m}^{[k]}+h u_{m}^{[k]}+r_{m}^{[k-1]}+\mathcal{O}\left(h^{M+2}\right),
\end{aligned}
$$

where $r_{m}^{[k-1]}=\sum_{i=1}^{M} \frac{h^{i+1}}{(i+1) !} \frac{d^{i}}{d t^{i}} F\left(t_{m}, e_{m}^{[k-1]}\right)$ and $u_{m}^{[k]}=f\left(t_{m}, y_{m}\right)-f\left(t_{m}, \eta_{m}^{[k-1]}+\right.$ $\left.\delta_{m}^{[k]}\right)$. Taylor expanding $f\left(t_{m}, \eta_{m}^{[k-1]}+\delta_{m}^{[k]}\right)$ about $y=y_{m}$ gives

$$
u_{m}^{[k]}=\sum_{i=1}^{S-2} \frac{(-1)^{i+1}}{i !} f_{y^{i}}\left(t_{m}, y_{m}\right)\left(e_{m}^{[k]}\right)^{i}+\mathcal{O}\left(\left(e_{m}^{[k]}\right)^{S-1}\right) .
$$


We will also need a Taylor expansion of $F\left(t, e^{(k-1)}(t)\right)=f(t, y(t))-f(t, y(t)-$ $\left.e^{(k-1)}(t)\right)$,

$$
F\left(t, e^{(k-1)} t\right)=\sum_{j=1}^{S-2} \frac{(-1)^{j+1}}{j !} f_{y^{j}}(t, y(t))\left(e^{(k-1)}(t)\right)^{j}+\mathcal{O}\left(\left(e^{(k-1)}(t)\right)^{S-1}\right) .
$$

Since $\overrightarrow{\tilde{e}}^{[k-1]}$ has $(M-k+1)$ degrees of smoothness in the discrete sense, $\frac{d}{d t} \tilde{e}^{(k-1)}(t) \sim$ $\mathcal{O}(1)$ from Proposition 3.16. Thus,

$$
\begin{aligned}
\frac{d F}{d t}=\sum_{j=1}^{S-2} \frac{(-1)^{j+1} h^{k j}}{j !} & \left(\frac{d f_{y^{j}}}{d t}\left(\tilde{e}^{(k-1)}\right)^{j}+\frac{d\left(\tilde{e}^{(k-1)}\right)^{j}}{d t} f_{y^{j}}\right) \\
& +\mathcal{O}\left(h^{(k+1)(S-1)-1}\right) \sim \mathcal{O}\left(h^{k}\right) .
\end{aligned}
$$

We are now ready to bound $\left\|e^{[k]}\right\|_{\infty}$ by induction. By definition, $e_{0}^{[k]}=0$, so certainly, $e_{0}^{[k]} \sim \mathcal{O}\left(h^{k+2}\right)$. Assume that $e_{m}^{[k]} \sim \mathcal{O}\left(h^{k+2}\right)$. Then,

$$
\begin{aligned}
e_{m+1}^{[k]} & =e_{m}^{[k]}+h u_{m}^{[k]}+r_{m}^{[k-1]}+\mathcal{O}\left(h^{M+2}\right) \\
& \sim \mathcal{O}\left(h^{k+2}\right)+\mathcal{O}\left(h^{k+3}\right)+\mathcal{O}\left(h^{k+2}\right)+\mathcal{O}\left(h^{M+2}\right) \sim \mathcal{O}\left(h^{k+2}\right),
\end{aligned}
$$

since each term of $r_{m}^{[k-1]} \sim \mathcal{O}\left(h^{k+2}\right)$, and $u_{m}^{[k-1]}$ is bounded in (4.3). This completes the inductive proof for the error bound.

To prove the smoothness of the rescaled error vector, we use an inductive argument based on $s$, the degree of smoothness of $\overrightarrow{\tilde{e}}^{[k]}$. This is similar in spirit to the proof of Lemma 4.2. First, the rescaled error vector, $\overrightarrow{\tilde{e}}^{[k]}$, has at least 0 degrees of smoothness since $\left\|\overrightarrow{\tilde{e}}^{[k]}\right\|_{\infty} \sim \mathcal{O}(h)$ is bounded. Assume that $\overrightarrow{\tilde{e}}^{[k]}$ has $s<M-k$ degrees of smoothness. We will prove that $\overrightarrow{d_{1} \tilde{e}^{[k]}}$ has $s$ degrees of smoothness, from which we can conclude that $\overrightarrow{\tilde{e}}^{[k]}$ has $(s+1)$ degrees of smoothness in the discrete sense. The divided difference approximation to the derivative of the rescaled error vector can be expressed as

$$
\left(d_{1} \tilde{e}^{[k]}\right)_{m}=\frac{\tilde{e}_{m+1}^{[k]}-\tilde{e}_{m}^{[k]}}{h}=\tilde{u}_{m}^{[k]}+\tilde{r}_{m}^{[k-1]}+\mathcal{O}\left(h^{M-k}\right),
$$

where

$$
\tilde{u}_{m}^{[k]}=\sum_{i=1}^{S-2} \frac{(-1)^{i+1} h^{(k+1)(i-1)}}{i !} f_{y^{i}}\left(t_{m}, y_{m}\right)\left(\tilde{e}_{m}^{[k]}\right)^{i}+\mathcal{O}\left(h^{(S-2)(k+2)+1}\right)
$$

has $s$ degrees of smoothness, and

$$
\begin{array}{r}
\tilde{r}_{m}^{[k-1]}=\frac{r_{m}^{[k-1]}}{h^{k+2}}=\sum_{i=1}^{M} \frac{h^{i-1}}{(i+1) !} \frac{d^{i-1}}{d t^{i-1}}\left(\frac{1}{h^{k}} \frac{d}{d t} F\left(t_{m}, e^{(k-1)}\left(t_{m}\right)\right)\right) \\
=\sum_{i=1}^{M} \frac{h^{i-1}}{(i+1) !} \frac{d^{i-1}}{d t^{i-1}} \sum_{j=1}^{S-2} \frac{(-1)^{j+1}}{j !}( \\
\left(h^{k(j-1)} \frac{d f_{y^{j}}}{d t}\right)\left(\tilde{e}^{(k-1)}\right)^{j} \\
\left.+\frac{d\left(\tilde{e}^{(k-1)}\right)^{j}}{d t}\left(h^{k(j-1)} f_{y^{j}}\right)\right)
\end{array}
$$


has at least $\min (S-(j+1)+k(j-1)-1, M-k) \geq s$ degrees of smoothness. Since $\overrightarrow{d_{1} \tilde{e}^{[k]}}$ has $s$ degrees of smoothness, we can conclude that $\tilde{\tilde{e}}^{[k]}$ has $(M+1-k)$ degrees of smoothness.

Remark 4.4. For the case of non-uniformly distributed quadrature nodes, the rescaled error vector $\overrightarrow{\tilde{e}}^{[0]}$ possesses only one degree of smoothness. An example is given below. The lack of smoothness also holds for the rescaled error vector obtained after an RK prediction step in Section 5. Consider the case of four quadrature nodes distributed such that $h_{1}=\frac{H}{6}, h_{2}=\frac{2 H}{3}$ and $h_{3}=\frac{H}{6}$. Then, $\tilde{u}_{m}$ and $\frac{r_{m}}{h_{m}^{2}}$ in equation (4.1) only possess at most one degree of smoothness. For example, if $y(t)=t^{3}$, then

$$
\frac{r_{m}}{h_{m}^{2}}=\frac{1}{2 !} y^{(2)}\left(t_{m}\right)+\frac{h_{m}}{3 !} y^{(3)}\left(t_{m}\right)+\cdots
$$

has only one degree of discrete smoothness.

Remark 4.5. Theorem 4.1 can be extended to the case of non-uniformly distributed quadrature nodes, despite the reduced smoothness of the error vector. The proof is similar in spirit to that of Theorem 4.1, since the rescaled error vector only requires one degree of smoothness to show that $\left\|\vec{e}^{[k]}\right\| \sim \mathcal{O}\left(h^{k+2}\right)$. This robust behavior for the Euler case is in sharp contrast to the behavior of defect correction methods on a non-uniform grid, which show no increase in order at all. For related results, see [1].

\section{LOCAL ERROR OF IDC METHODS CONSTRUCTED WITH HIGH ORDER RK INTEGRATORS}

In this section, we first review properties of RK integrators, then provide the local error estimates for IDC methods which utilize high order RK methods in the prediction and correction steps. The outline for the error estimates is similar to the local error estimates for IDC methods constructed with forward Euler integrators, presented in the previous section. A key set of equations, (5.8), is derived for applying a high order RK correction. In the following theorems and lemmas, we will assume that the function $f(t, y)$ in IVP (2.1) has $S-1$ degrees of smoothness if the solution $y(t)$ has $S$ degrees of smoothness.

Definition 5.1. A $p$-stage explicit RK method can be expressed in the form

$$
\eta_{i+1}=\eta_{i}+\sum_{j=1}^{p} b_{j} k_{j}, \quad k_{j}=h f\left(t_{i}+c_{j} h, \eta_{i}+\sum_{l=1}^{j-1} a_{j l} k_{l}\right) \quad j=1,2,3, \ldots, p,
$$

where $a_{j l}, b_{j}$, and $c_{j}$ are real coefficients. An RK method is of order $r$ if $f(t, y)$ in (2.1) is sufficiently smooth and if $\left\|y\left(t_{i}+h\right)-\eta_{i+1}\right\| \leq K h^{r+1}$ for some constant $K>0$; i.e., the Taylor series expansion for the exact solution, $y\left(t_{i}+h\right)$, and $\eta_{i+1}$ coincide up to and including the term $h^{r}$.

Proposition 5.2. Let $y(t)$, the solution to IVP (2.1), have $S \geq r$ degrees of smoothness, and let $\eta$ be the numerical solution obtained using an $r^{\text {th }}$ order $R K$ method. If $t_{m+1}-t_{m}=h$, then

$$
\eta_{m+1}=\eta_{m}+\sum_{j=}^{S-1} \frac{h^{j}}{j !} E_{j}\left(t_{m}, \eta_{m}\right)+R_{r}\left(t_{m}, \eta_{m}\right)+\mathcal{O}\left(h^{S}\right),
$$


where the functions $E_{j}(t, y)$ are the elementary differentials of $y$,

$$
y^{(j)}(t):=E_{j}(t, y(t))=\sum_{q_{y}^{i}+q_{t}^{i} \leq j-1} \alpha_{q_{y}^{1} q_{t}^{1} \cdots w_{n_{i}} w_{f}}^{w_{1} q_{t}^{n_{i}}} \prod_{i=1}^{n_{i}}\left(f_{t^{q_{t}^{i}} y^{q_{y}^{i}}}\right)^{w_{i}} f^{w_{f}},
$$

and the remainder term,

$R_{r}(t, \eta)=\sum_{j=r+1}^{S-1} h^{j}\left(\sum_{q_{y}^{i}+q_{t}^{i} \leq j-1} \beta_{q_{t}^{1} q_{y}^{1} \cdots q_{t}^{n_{i}} q_{y}^{n_{i}} q^{f}}^{w_{1} w_{2} \cdots w_{n_{i}} w_{f}} \prod_{i=1}^{n_{i}}\left(f_{t^{q_{t}^{i}} y^{q_{y}^{i}}}\right)^{w_{i}}\left(t_{m}, \eta_{m}\right) f^{w_{f}}\left(t_{m}, \eta_{m}\right)\right)$,

has constant coefficients $\beta_{q_{y}^{1} q_{t}^{1} \cdots q_{y}^{n_{i}} q_{t}^{n_{i}}}^{w_{1} w_{i} w_{n_{1}} w_{f}}$ determined by the specific RK method.

Proof. Equation (5.1) comes from a Taylor expansion of $\eta_{m+1}$ about $t=t_{m}$. Note that the first $r+1$ terms coincide with the Taylor expansion of the exact solution,

$$
y\left(t_{m+1}\right)=y\left(t_{m}\right)+\sum_{j=1}^{S-1} \frac{h^{j}}{j !} y^{(j)}\left(t_{m}\right)+\mathcal{O}\left(h^{S}\right) .
$$

The remainder term can be proved by induction. Once again, the range of summation, $q_{t}^{i}+q_{y}^{i} \leq j-1$, is not restrictive since some of the coefficients $\beta_{q_{y}^{1} q_{t}^{1} \ldots q_{y}^{n_{n}} q_{t}^{n_{i}}}^{w_{1} w_{2} w_{f}}$ will be zero.

Theorem 5.3. Let $y(t)$, the solution to the IVP (2.1), have at least $S \geq M+2$ degrees of smoothness in the continuous sense. Then, the local error for an IDC method constructed using $(M+1)$ uniformly distributed nodes $\left(t_{m}=m h, m=\right.$ $0, \ldots, M)$, an $\left(r_{0}\right)^{t h}$ order $R K$ method in the prediction step and $\left(r_{1}, r_{2}, \ldots, r_{k_{l}}\right)^{t h}$ order RK methods is $\mathcal{O}\left(h^{\left(s_{k_{l}}+1\right)}\right)$, where $s_{k_{l}}=\sum_{j=0}^{k_{l}} r_{j} \leq M+1$.

Proof. The proof of Theorem 5.3 follows from two lemmas discussed in this section. We will show in Lemma 5.4 that the theorem holds for $k=0$, and in Lemma 5.5 that an inductive argument is satisfied.

Lemma 5.4 (prediction step). Consider an IDC method constructed using $(M+1)$ uniformly distributed nodes, and an $r_{0}^{\text {th }}$ order $R K$ method for the prediction step. Let $y(t)$, the solution to IVP (2.1), have at least $S \geq M+2$ degrees of smoothness in the continuous sense, and let $\left(\eta_{0}^{[0]}, \ldots, \eta_{m}^{[0]}, \ldots, \eta_{M}^{[0]}\right)$ be the numerical solution computed after the prediction step. Then, the error vector, $\vec{e}^{[0]}=\vec{y}-\vec{\eta}^{[0]}$, satisfies $\left\|\vec{e}^{[0]}\right\|_{\infty} \sim$ $\mathcal{O}\left(h^{r_{0}+1}\right)$, and the rescaled error vector, $\overrightarrow{\tilde{e}}^{[0]}=\frac{1}{h^{r_{0}}} \vec{e}^{[0]}$, has $\min \left(S-r_{0}, M\right)$ degrees of smoothness in the discrete sense.

Proof. We drop the superscript [0] since there is no ambiguity. First, note that a Taylor expansion of the error, $e_{m+1}=y_{m+1}-\eta_{m+1}$, about $t=t_{m}$ (see equations (5.3) and (5.1) ), results in

$$
e_{m+1}=e_{m}+u_{m}+r_{1, m}-r_{2, m}+\mathcal{O}\left(h^{S}\right),
$$


where

$$
\begin{aligned}
& u_{m}=\sum_{i=1}^{r_{0}} \frac{h^{i}}{i !}\left(E_{i}\left(t_{m}, y_{m}\right)-E_{i}\left(t_{m}, \eta_{m}\right)\right) \\
&=\sum_{i=1}^{r_{0}} \frac{h^{i}}{i !} \sum_{j=1}^{S-1-i} \frac{(-1)^{j-1}\left(e_{m}\right)^{j}}{j !} \frac{\partial^{j} E_{i}}{\partial y^{j}}\left(t_{m}, y_{m}\right)+\mathcal{O}\left(\left(e_{m}\right)^{S-i}\right), \\
& r_{1, m}=\sum_{i=r_{0}+1}^{S-1} \frac{h^{i}}{i !} y^{(i)}\left(t_{m}\right), \\
& r_{2, m}=\sum_{j=r_{0}+1}^{S-1} h^{j} \sum_{t}^{i}+q_{y}^{i} \leq j-1 \\
& \beta_{q_{t}^{1} q_{y}^{1} \cdots q_{t}^{n_{i}} q_{y}^{n_{i}} q^{f}}^{w_{1} w_{2} \cdots w_{n_{i}} w_{f}} \prod_{i=1}^{n_{i}}\left(f_{t^{q_{t}^{i}} y^{q_{y}^{i}}}\right)^{w_{i}}\left(t_{m}, \eta_{m}\right) f^{w_{f}}\left(t_{m}, \eta_{m}\right) .
\end{aligned}
$$

We are now ready to bound $\left\|\vec{e}^{[0]}\right\|_{\infty}$ by induction. By definition, $e_{0}=0$, so certainly, $e_{0} \sim\left(h^{r_{0}+1}\right)$. Assume that $e_{m} \sim \mathcal{O}\left(h^{r_{0}+1}\right)$. Since $u_{m} \sim \mathcal{O}\left(h^{r_{0}+2}\right), r_{1, m} \sim \mathcal{O}\left(h^{r_{0}+1}\right)$ and $r_{2, m} \sim \mathcal{O}\left(h^{r_{0}+1}\right)$, we have $e_{m+1} \sim \mathcal{O}\left(h^{r_{0}+1}\right)$, which completes the inductive proof.

To prove the smoothness of the rescaled error vector, we will again use an inductive approach, but this time, with respect to $s$, the degree of smoothness. First, note that a divided difference approximation to the derivative of the rescaled error vector gives

$$
\left(d_{1} \tilde{e}\right)_{m}=\frac{\tilde{e}_{m+1}-\tilde{e}_{m}}{h}=\tilde{u}_{m}+\tilde{r}_{1, m}-\tilde{r}_{2, m}+\mathcal{O}\left(h^{S-r_{0}-1}\right)
$$

where

$$
\begin{aligned}
\tilde{u}_{m} & =\frac{u_{m}}{h^{r_{0}+1}}=\sum_{i=1}^{r_{0}} \frac{1}{i !} \sum_{j=1}^{S-1-i} \frac{(-1)^{j-1} h^{i+r_{0}(j-1)-1}}{j !} \frac{\partial^{j} E_{i}}{\partial y^{j}}\left(t_{m}, y_{m}\right)\left(\tilde{e}_{m}\right)^{j}+\mathcal{O}\left(h^{S-r_{0}-1}\right), \\
\tilde{r}_{1, m} & =\frac{r_{1, m}}{h^{r_{0}+1}}=\sum_{i=r_{0}+1}^{S-1} \frac{h^{i-r_{0}-1}}{i !} y^{(i)}\left(t_{m}\right), \\
\tilde{r}_{2, m}= & \frac{R_{r_{0}}\left(t_{m}, \eta_{m}\right)}{h^{r_{0}+1}} \\
& =\sum_{j=0}^{S-r_{0}-2} h^{j} \sum_{q_{t}^{i}+q_{y}^{i} \leq j+r_{0}} \beta_{q_{t}^{1} q_{y}^{1} \cdots q_{t}^{n_{i}} q_{y}^{n_{i}} q^{f}}^{w_{1} w_{2} \cdots w_{n_{i}} w_{f}} \prod_{i=1}^{n_{i}}\left(f_{t^{q_{t}^{i}} y^{q_{y}^{i}}}\right)^{w_{i}}\left(t_{m}, \eta_{m}\right) f^{w_{f}}\left(t_{m}, \eta_{m}\right) .
\end{aligned}
$$

We are now ready to prove that $\overrightarrow{\tilde{e}}$ has $\min \left(S-r_{0}, M\right)$ degrees of smoothness by induction. Since $\|\overrightarrow{\tilde{e}}\|_{\infty} \sim \mathcal{O}(h)$ is bounded, $\overrightarrow{\tilde{e}}$ has at least zero degrees of smoothness in the discrete sense. Assume that $\overrightarrow{\tilde{e}}$ has $s<\min \left(S-r_{0}, M\right)$ degrees of smoothness in the discrete sense. We will prove that $\overrightarrow{d_{1} \tilde{e}}$ has $s$ degrees of smoothness, from which we can conclude that $\overrightarrow{\tilde{e}}$ has $(s+1)$ degrees of smoothness in the discrete sense. From a similar argument as in the proof of Lemma 4.2, $\overrightarrow{\tilde{u}}$ has $s$ degrees of smoothness in the discrete sense. Assuming that $y(t)$ has $S$ degrees of smoothness, $\vec{r}_{1}$ has $\left(S-r_{0}-1\right)$ degrees of smoothness. Since $\overrightarrow{\tilde{e}}$ has $s$ degrees of smoothness, $\vec{e}$ has $\min \left(s+r_{0}, M\right)$ degrees of smoothness and $\vec{\eta}=\vec{y}-\vec{e}$ has $\min \left(s+r_{0}, M\right)$ degrees of smoothness. Thus, $\overrightarrow{\tilde{r}_{2}}$ has $\min \left(s+r_{0}, S-r_{0}-1, M\right)$, at least $s$, degrees of smoothness in the discrete sense. Therefore $\overrightarrow{\tilde{e}}$ has $(s+1)$ degrees of smoothness. 
We can now conclude that $\overrightarrow{\tilde{e}}$ has $\min \left(S-r_{0}, M\right)$ degrees of smoothness in the discrete sense.

Lemma 5.5 (correction step). Let $y(t)$, the solution to IVP (2.1), have at least $S \geq M+2$ degrees of smoothness in the continuous sense. Consider an IDC method, constructed using $(M+1)$ uniformly distributed nodes, an $\left(r_{0}\right)^{\text {th }}$ order $R K$ method in the prediction step and $\left(r_{1}, r_{2}, \ldots, r_{k_{l}}\right)^{\text {th }}$ order $R K$ methods in the respective correction loops. If the error vector after the $(k-1)^{\text {st }}$ loop satisfies $\vec{e}^{[k-1]} \sim \mathcal{O}\left(h^{s_{k-1}+1}\right)$, and the rescaled error vector, $\overrightarrow{\tilde{e}}^{[k-1]}=\frac{1}{h^{s_{k-1}}} \vec{e}^{[k-1]}$, has $(M+$ $\left.1-s_{k-1}\right)$ degrees of smoothness in the discrete sense, then, the updated error vector, $\vec{e}^{[k]}$, satisfies $\left\|\vec{e}^{[k]}\right\|_{\infty} \sim \mathcal{O}\left(h^{s_{k}+1}\right)$, and the rescaled error vector, $\overrightarrow{\tilde{e}}^{[k]}=\frac{1}{h^{s_{k}}} \vec{e}^{[k]}$, has $\left(M+1-s_{k}\right)$ degrees of smoothness in the discrete sense.

The proof of this lemma is very technical and involved. First, we discuss properties of the error function, $e^{(k-1)}(t)$. Then, the actual construction of IDC methods using RK integrators within the correction loops is described. A discussion of the properties of the numerical error vector is given before Lemma 5.5 is proved. Note that for a non-uniform distribution of nodes, $\overrightarrow{\tilde{e}}^{[0]}$ has only one degree of smoothness (as discussed in Remark 4.4); hence, Lemma 5.5 does not apply.

5.1. Properties of the error function. We write the error equation (2.4) as

$$
\left(Q^{(k-1)}\right)^{\prime}(t)=F\left(t, Q^{(k-1)}(t)-E^{(k-1)}(t)\right) \doteq G^{(k-1)}\left(t, Q^{(k-1)}(t)\right),
$$

where $Q^{(k-1)}(t)=e^{(k-1)}(t)+E^{(k-1)}(t)$ and $E^{(k-1)}(t)=\int_{0}^{t} \epsilon^{(k-1)}(\tau) d \tau$. Our analysis for the error vector will rely on this form of error equation, (5.4), or the scaled variant,

$$
\left(\tilde{Q}^{(k-1)}\right)^{\prime}(t)=\tilde{G}^{(k-1)}\left(t, \tilde{Q}^{(k-1)}(t)\right),
$$

where $\tilde{Q}^{(k-1)}(t)=\frac{Q^{(k-1)}(t)}{h^{s} k-1}$ and $\tilde{G}^{(k-1)}\left(t, \tilde{Q}^{(k-1)}(t)\right)=\frac{G^{(k-1)}\left(t, h^{s k-1} \tilde{Q}^{(k-1)}(t)\right)}{h^{s k-1}}$.

Proposition 5.6. If $e^{(k-1)}(t) \sim \mathcal{O}\left(h^{s_{k-1}+1}\right)$, then $Q^{(k-1)}(t) \sim \mathcal{O}\left(h^{s_{k-1}+1}\right)$ and $G^{(k-1)}\left(t, Q^{(k-1)}(t)\right) \sim \mathcal{O}\left(h^{s_{k-1}+1}\right)$.

Proof. From the above definition of $G$,

$$
\begin{aligned}
G^{(k-1)}(t & \left., Q^{(k-1)}(t)\right)=F\left(t, e^{(k-1)}(t)\right) \\
& =\sum_{i=1}^{S-2} \frac{(-1)^{i+1}}{i !} \frac{\partial^{i} f}{\partial y^{i}}(t, y(t))\left(e^{(k-1)}(t)\right)^{i}+\mathcal{O}\left(h^{\left(s_{k-1}+1\right)(S-1)}\right) \sim \mathcal{O}\left(h^{s_{k-1}+1}\right)
\end{aligned}
$$

and $Q^{(k-1)}(t)=\int_{0}^{t} G^{(k-1)}\left(\tau, Q^{(k-1)}(\tau)\right) d \tau \sim \mathcal{O}\left(h^{s_{k-1}+1}\right)$.

Proposition 5.7. Suppose $\vec{e}^{[k-1]} \sim \mathcal{O}\left(h^{s_{k-1}+1}\right)$ and the rescaled error vector, $\tilde{\tilde{e}}^{[k-1]}$, has $\left(M+1-s_{k-1}\right)$ degrees of smoothness in the discrete sense. Then

$$
\frac{d^{l}}{d t^{l}} \tilde{Q}^{(k-1)}(t) \sim \mathcal{O}(1) \text { if } \quad l \leq M+1-s_{k-1}, \quad \forall t \in[0, H] .
$$


Proof. Since the rescaled error vector, $\overrightarrow{\tilde{e}}^{[k-1]}$, has $\left(M+1-s_{k-1}\right)$ degrees of smoothness in the discrete sense, from Proposition [3.16, $\frac{d^{l}}{d t^{l}} \tilde{e}^{(k-1)}(t) \sim \mathcal{O}(1)$, for $l \leq$ $M+1-s_{k-1}, \forall t \in[0, H]$. From Proposition 5.6.

$$
\begin{aligned}
Q^{(k-1)}(t) & =\int_{0}^{t} G^{(k-1)}\left(\tau, Q^{(k-1)}(\tau)\right) d \tau \\
& =\int_{0}^{t} \sum_{i=1}^{S-2} \frac{(-1)^{i+1}}{i !} \frac{\partial^{i} f}{\partial y^{i}}(\tau, y(\tau))\left(e^{(k-1)}(\tau)\right)^{i} d \tau+\mathcal{O}\left(h^{\left(s_{k-1}+1\right)(S-1)+1}\right),
\end{aligned}
$$

we have

$$
\begin{aligned}
\frac{d}{d t} \tilde{Q}^{(k-1)}(t) & =\sum_{i=1}^{S-2} \frac{(-1)^{i+1}}{i !} h^{s_{k-1}(i-1)} \frac{\partial^{i} f}{\partial y^{i}}(t, y(t))\left(\tilde{e}^{(k-1)}(t)\right)^{i}+\mathcal{O}\left(h^{\left(s_{k-1}+1\right)(S-2)+1}\right) \\
& \sim \mathcal{O}(1) \\
\frac{d^{l}}{d t^{l}} \tilde{Q}^{(k-1)}(t) & \sim \frac{d^{l-1}}{d t^{l-1}}\left(\tilde{e}^{(k-1)}(t)\right) \sim \mathcal{O}(1), \quad \text { for } \quad l=2, \ldots, M+1-s_{k-1} .
\end{aligned}
$$

Note that if $\overrightarrow{\tilde{e}}^{[k-1]}$ does not have the required degrees of smoothness (e.g., if a nonuniform mesh is used to construct the IDC method), then the discrete derivative of $\frac{d^{l}}{d t^{l}} \tilde{Q}^{(k-1)}$ is no longer $\mathcal{O}(1)$.

Proposition 5.8. $\tilde{Q}^{(k-1)}(t)$ satisfies

$$
\tilde{Q}_{m+1}^{[k-1]}=\tilde{Q}_{m}^{[k-1]}+\sum_{i=1}^{M-s_{k-1}} \frac{h^{i}}{i !} \tilde{G}_{i-1}^{(k-1)}\left(t_{m}, \tilde{Q}_{m}^{[k-1]}\right)+\mathcal{O}\left(h^{M+1-s_{k-1}}\right)
$$

or, equivalently,

$$
\begin{aligned}
e_{m+1}^{[k-1]}= & e_{m}^{[k-1]}+h^{s_{k-1}} \sum_{i=1}^{M-s_{k-1}} \frac{h^{i}}{i !} \tilde{G}_{i-1}^{(k-1)}\left(t_{m}, \tilde{Q}_{m}^{[k-1]}\right)+\mathcal{O}\left(h^{M+1}\right) \\
& -\int_{t_{m}}^{t_{m+1}} \epsilon^{(k-1)}(\tau) d \tau
\end{aligned}
$$

where

$$
\tilde{G}_{i-1}^{(k-1)}(t, \tilde{Q}):=\sum_{q_{t}^{i}+q_{\tilde{Q}}^{i} \leq i-1} \gamma_{q_{t}^{1} q_{\tilde{Q}}^{1} \cdots q_{t}^{n_{i}} q_{\tilde{Q}}^{n_{i}}}^{w_{1} \cdots w_{n_{i}} w_{f}} \prod_{i=1}^{n_{i}}\left(\tilde{G}_{t^{q_{t}^{i}} \tilde{Q}^{q_{\tilde{Q}}^{i}}}^{(k-1)}\right)^{w_{i}}\left(\tilde{G}^{(k-1)}\right)^{w_{f}}, \quad i \leq M-s_{k-1} .
$$

As before, $\gamma_{q_{t}^{1} q_{\tilde{Q}}^{1} \cdots q_{t}^{n_{i}} q_{\tilde{Q}}^{n_{i}}}^{w_{1} \ldots w_{n_{i}} w_{f}}$ are constant coefficients, and $w_{i}, w_{f}, q_{t}^{i}, q_{\tilde{Q}}^{i}$ and $n_{i}$ are non-negative integers. Note that

$$
\tilde{G}_{i-1}^{(k-1)}\left(t, \tilde{Q}^{(k-1)}(t)\right):=\frac{d^{i-1} \tilde{G}^{(k-1)}}{d t^{i-1}}=\frac{d^{i}}{d t^{i}} \tilde{Q}^{(k-1)}(t) \sim \mathcal{O}_{h}(1), \quad i \leq M-s_{k-1} .
$$


5.2. RK integrators within the correction loop of IDC methods. A $p$-stage, $r^{\text {th }}$ order RK method applied to (5.5) gives

$$
\begin{aligned}
\tilde{k}_{1} & =\tilde{G}^{(k-1)}\left(t_{0}, \tilde{Q}_{0}^{[k-1]}\right), \\
\tilde{k}_{2} & =\tilde{G}^{(k-1)}\left(t_{0}+c_{2} h,\left(\tilde{Q}_{0}^{[k-1]}+h a_{2,1} \tilde{k}_{1}\right)\right), \\
& \ldots \\
\tilde{k}_{p} & =\tilde{G}^{(k-1)}\left(t_{0}+c_{p} h,\left(\tilde{Q}_{0}^{[k-1]}+h\left(a_{p, 1} \tilde{k}_{1}+\ldots+a_{p, p-1} \tilde{k}_{p-1}\right)\right)\right), \\
\tilde{\Omega}_{1}^{[k]} & =\tilde{Q}_{0}^{[k-1]}+h\left(b_{1} \tilde{k}_{1}+\cdots+b_{p} \tilde{k}_{p}\right),
\end{aligned}
$$

where $\Omega$ denotes the solution in numerical space. In the actual implementation, we discretize the error equation (2.4) as follows:

$$
\begin{aligned}
k_{1} & =F\left(t_{0}, e_{0}^{[k-1]}\right), \\
k_{2} & =F\left(t_{0}+c_{2} h, e_{0}^{[k-1]}+h a_{2,1} k_{1}-\int_{t_{0}}^{t_{0}+c_{2} h} \epsilon^{(k-1)}(\tau) d \tau\right), \\
& \ldots \\
k_{p} & =F\left(t_{0}+c_{p} h, e_{0}^{[k-1]}+h \sum_{i=1}^{p} a_{p i} k_{i}-\int_{t_{0}}^{t_{0}+c_{p} h} \epsilon^{(k-1)}(\tau) d \tau\right), \\
\delta_{1}^{[k]} & =e_{0}^{[k-1]}+h \sum_{i=1}^{t_{0}+h} b_{i} k_{i}-\int_{t_{0}}^{t^{(k-1)}}(\tau) d \tau .
\end{aligned}
$$

Proposition 5.9. The Taylor series for $\tilde{e}^{(k-1)}\left(t_{0}+h\right)=\frac{1}{h^{s} k-1} e^{(k-1)}\left(t_{0}+h\right)$ and for $\frac{1}{h^{s} k-1} \delta_{1}^{[k]}$ above coincide up to and including the term $h^{r}$, for a sufficiently smooth error function $\tilde{e}^{(k-1)}(t)$.

Proof. First, we prove by induction that

$$
h^{s_{k-1}} \tilde{k}_{i}=k_{i}, \quad \forall i=1, \ldots, p .
$$

Since

$$
h^{s_{k-1}} \tilde{k}_{1}=G^{(k-1)}\left(t_{0}, Q_{0}^{[k-1]}\right)=F\left(t_{0}, e^{(k-1)}\left(t_{0}\right)\right)=k_{1},
$$

the claim is true for $i=1$. Assume that (5.9) is true for $1, \ldots, i$. Then,

$$
\begin{aligned}
& h^{s_{k-1}} \tilde{k}_{i+1}=G^{(k-1)}\left(t_{0}+c_{i+1} h, h^{s_{k-1}}\left(\tilde{Q}^{(k-1)}\left(t_{0}\right)+h \sum_{j=1}^{i} a_{i+1, j} \tilde{k}_{j}\right)\right) \\
& =G^{(k-1)}\left(t_{0}+c_{i+1} h, Q^{(k-1)}\left(t_{0}\right)+h \sum_{j=1}^{i} a_{i+1, j} k_{j}\right) \\
& =F\left(t_{0}+c_{i+1} h, Q^{(k-1)}\left(t_{0}\right)+h \sum_{j=1}^{i} a_{i+1, j} k_{j}-E^{(k-1)}\left(t_{0}+c_{i+1} h\right)\right) \\
& =F\left(t_{0}+c_{i+1} h, e_{0}^{[k-1]}+h \sum_{j=1}^{i} a_{i+1, j} k_{j}-\int_{t_{0}}^{t_{0}+c_{i+1} h} \epsilon^{(k-1)}(\tau) d \tau\right)=k_{i+1},
\end{aligned}
$$


which completes the inductive proof. Also,

$$
\begin{aligned}
\delta_{1}^{[k]} & :=h^{s_{k-1}} \tilde{\Omega}_{1}^{[k]}-E\left(t_{1}\right) \\
& =Q^{(k-1)}\left(t_{0}\right)-E\left(t_{1}\right)+h\left(b_{1} k_{1}+\cdots+b_{p} k_{p}\right) \\
& =e_{0}^{[k-1]}+h\left(b_{1} k_{1}+\cdots+b_{p} k_{p}\right)-\int_{t_{0}}^{t_{0}+h} \epsilon^{(k-1)}(\tau) d \tau .
\end{aligned}
$$

Since the Taylor series for $\tilde{Q}^{(k-1)}\left(t_{0}+h\right)$ and $\tilde{\Omega}_{1}^{[k]}$ coincide, up to and including the term $h^{r}$, the Taylor series for $\frac{1}{h^{s} k-1} e^{(k-1)}\left(t_{0}+h\right)$ and $\frac{1}{h^{s} k-1} \delta_{1}^{[k]}$ also coincide, up to and including the term $h^{r}$.

Proposition 5.10. Suppose that the error equation (2.4) is discretized using the algorithm in (5.8). Then, the numerical error vector satisfies

$$
\begin{aligned}
\delta_{m+1}^{[k]}=\delta_{m}^{[k]} & +\sum_{i=1}^{r_{k}} \frac{h^{s_{k-1}+i}}{i !} \tilde{G}^{(k-1)}\left(t_{m}, \tilde{\Omega}_{m}^{[k]}\right)+h^{s_{k-1}} \tilde{R}_{r_{k}+1}\left(t_{m}, \tilde{\Omega}_{m}^{[k]}\right) \\
& -\int_{t_{m}}^{t_{m+1}} \epsilon^{(k-1)}(\tau) d \tau+\mathcal{O}\left(h^{M+1}\right)
\end{aligned}
$$

where $\tilde{\Omega}_{m}^{[k]}=\frac{1}{h^{s_{k}-1}}\left(\delta^{[k]}\left(t_{m}\right)+\int_{0}^{t_{m}} \epsilon^{(k-1)}(\tau) d \tau\right)$ and

$$
\tilde{R}_{r_{k}+1}(t, \tilde{\Omega})=\sum_{q=r_{k}+1}^{M-s_{k-1}} h^{q} \sum_{q_{t}^{i}+q_{\tilde{Q}}^{i} \leq q-1} \zeta_{q_{t}^{1} q_{\tilde{Q}}^{1} \cdots q_{t}^{n_{i}} q_{\tilde{Q}}^{n_{n}}}^{w_{1} w_{2} \cdots w_{n_{i}} w_{f}} \prod_{i=1}^{n_{i}}\left(\tilde{G}^{(k-1)}(t, \tilde{\Omega})\right)^{w_{i}}\left(\tilde{G}^{(k-1)}(t, \tilde{\Omega})\right)^{w_{f}}
$$

Here $\zeta_{q_{t}^{1} q_{\tilde{Q}}^{1} \cdots q_{t}^{n_{i}} q_{\tilde{Q}}^{n_{i}}}^{w_{1} w_{2} \cdots w_{n_{1}} w_{f}}$ are constant coefficients determined by (5.8). Note that the $\mathcal{O}\left(h^{M+1}\right)$ truncation error arises from numerically computing $\int_{t_{m}}^{t_{m+1}} \epsilon^{(k-1)}(\tau) d \tau$, and that $\tilde{G}_{t^{q_{t}^{i}} \tilde{Q}^{q_{\tilde{Q}}^{i}}}^{(k-1)}(t, \tilde{\Omega}) \sim \mathcal{O}_{h}(1)$ for $q_{t}^{i}+q_{\tilde{Q}}^{i} \leq M-s_{k-1}$. We omit the proof, which is similar in spirit to the proof of Proposition 5.2

Remark 5.11. In algorithm (5.8), an evaluation of $f(t, \eta(t))$ is needed at intermediate stages, e.g., at $t=t_{0}+c_{p} h$; this results in additional function evaluations of $f(t, \eta(t))$. Since function evaluations are usually the most computationally intensive portion of an algorithm, we perform polynomial interpolations from known values instead. Specifically, given $\vec{f}=\left(f\left(t_{0}, \eta_{0}\right), \ldots, f\left(t_{m}, \eta_{m}\right), \ldots, f\left(t_{M}, \eta_{M}\right)\right)$, an $M^{t h}$ degree Lagrange interpolant, $L^{M}(t, f)$, see (3.2), is constructed and used to approximate $\left.f(t, \eta(t))\right|_{t=t_{0}+c_{p} h}$. This approximation has truncation error, $\mathcal{O}\left(h^{M+2}\right)$. 
Remark 5.12. To evaluate the integral term in algorithm (5.8), we integrate the corresponding Lagrange interpolant, $\int_{t_{0}}^{t_{0}+c_{i} h} L^{M}\left(t, \epsilon^{(k-1)}\right) d t, i=1, \ldots, p$, with truncation error, $\mathcal{O}\left(h^{M+2}\right)$.

Proof of Lemma 5.5. Subtracting the numerical error vector, (5.10), from the integrated error equation, (5.7),

$$
\begin{aligned}
e_{m+1}^{[k]}= & e_{m+1}^{[k-1]}-\delta_{m+1}^{[k]} \\
= & e_{m}^{[k]}+h^{s_{k-1}} \sum_{i=r_{k}+1}^{M+1-s_{k-1}} \frac{h^{i}}{i !} \tilde{G}_{i-1}^{(k-1)}\left(t_{m}, \tilde{Q}_{m}^{[k-1]}\right) \\
& +h^{s_{k-1}} \sum_{i=1}^{r_{k}} \frac{h^{i}}{i !}\left(\tilde{G}_{i-1}^{(k-1)}\left(t_{m}, \frac{e_{m}^{[k-1]}+E^{(k-1)}\left(t_{m}\right)}{h^{s_{k-1}}}\right)\right. \\
& \left.-\tilde{G}_{i-1}^{(k-1)}\left(t_{m}, \frac{\delta_{m}^{[k]}+E^{(k-1)}\left(t_{m}\right)}{h^{s_{k-1}}}\right)\right) \\
& -h^{s_{k-1}} \tilde{R}_{r_{k}+1}\left(t_{m}, \frac{\delta_{m}^{[k]}+E^{(k-1)}\left(t_{m}\right)}{h^{s_{k-1}}}\right)+\mathcal{O}\left(h^{M+2}\right) \\
= & e_{m}^{[k]}+u_{m}^{[k]}+r_{1, m}^{[k-1]}-r_{2, m}^{[k]}+\mathcal{O}\left(h^{M+2}\right),
\end{aligned}
$$

where

$$
\begin{aligned}
u_{m}^{[k]}= & h^{s_{k-1}} \sum_{i=1}^{r_{k}} \frac{h^{i}}{i !}\left(\tilde{G}_{i-1}\left(t_{m}, \frac{e_{m}^{[k-1]}+E^{(k-1)}\left(t_{m}\right)}{h^{s_{k-1}}}\right)\right. \\
& \left.-\tilde{G}_{i-1}\left(t_{m}, \frac{\delta_{m}^{[k]}+E^{(k-1)}\left(t_{m}\right)}{h^{s_{k-1}}}\right)\right) \\
= & \sum_{i=1}^{r_{k}} \frac{h^{i}}{i !} \frac{d^{i-1}}{d t^{i-1}}\left(G^{(k-1)}\left(t_{m}, e_{m}^{[k-1]}+E^{(k-1)}\left(t_{m}\right)\right)\right. \\
& \left.-G^{(k-1)}\left(t_{m}, \delta_{m}^{[k]}+E^{(k-1)}\left(t_{m}\right)\right)\right) \\
= & \sum_{i=1}^{r_{k}} \frac{h^{i}}{i !} \frac{d^{i-1}}{d t^{i-1}}\left(f\left(t_{m}, y\left(t_{m}\right)\right)-f\left(t_{m}, \eta^{(k)}\left(t_{m}\right)\right)\right) \\
= & \sum_{i=1}^{r_{k}} \frac{h}{i !} h^{i-1} \frac{d^{i-1}}{d t^{i-1}}\left(\sum_{j=1}^{S-2} \frac{(-1)^{j+1}}{j !} \frac{\partial^{j} f}{\partial y^{j}}\left(t_{m}, y\left(t_{m}\right)\right)\left(e_{m}^{[k]}\right)^{j}+\mathcal{O}\left(\left(e_{m}^{[k]}\right)^{S-1}\right)\right),
\end{aligned}
$$

and since $s_{k}=s_{k-1}+r_{k}$, 


$$
\begin{gathered}
r_{1, m}^{[k-1]}=h^{s_{k-1}} \sum_{i=r_{k}+1}^{M+1-s_{k-1}} \frac{h^{i}}{i !} \tilde{G}_{i-1}\left(t_{m}, \tilde{Q}_{m}^{[k-1]}\right) \\
=h^{s_{k}+1} \sum_{i=0}^{M-s_{k}} \frac{1}{\left(r_{k}+i+1\right) !} h^{i} \frac{d^{i}}{d t^{i}}\left(\frac{d^{r_{k}}}{d t^{r_{k}}} \tilde{G}\left(t_{m}, \tilde{Q}_{m}^{[k-1]}\right)\right), \\
r_{2, m}^{[k]}=h^{s_{k-1}} \tilde{R}_{r_{k}+1}\left(t_{m}, \frac{\delta_{m}^{[k]}+E^{(k-1)}\left(t_{m}\right)}{h^{s_{k-1}}}\right) \\
=h^{s_{k}+1} \sum_{i=0}^{M+1-s_{k}} h^{i} \sum_{q_{\tilde{Q}}^{i}+q_{t}^{i} \leq i+r_{k}} \zeta_{q_{\tilde{Q}}^{1} q_{t}^{1} \cdots q_{\tilde{Q}_{n}}^{w_{i}} q_{t}^{n_{i}}}^{w_{i} w_{i}} \prod_{i=1}^{n_{i}}\left(\tilde{G}_{\tilde{Q}^{q_{\tilde{Q}}} t_{t^{q_{t}^{i}}}}\left(t, \tilde{\Omega}_{m}^{[k]}\right)\right)^{w_{i}} \tilde{G}^{w_{f}}\left(t, \tilde{\Omega}_{m}^{[k]}\right)
\end{gathered}
$$

We are now ready to bound $\left\|e^{[k-1]}\right\|_{\infty}$ using an inductive argument. By definition, $e_{0}^{[k]}=0$, so certainly $e_{0}^{[k]} \sim \mathcal{O}\left(h^{s_{k}+1}\right)$. Assume that $e_{m}^{[k]} \sim \mathcal{O}\left(h^{s_{k}+1}\right)$. From (5.12), $u_{m}^{[k]} \sim \mathcal{O}\left(h^{s_{k}+2}\right)$. By Proposition 5.7 $\frac{d^{r_{k}}}{d t^{r_{k}}} \tilde{G}\left(t_{m}, \tilde{Q}_{m}^{[k-1]}\right)=\left.\frac{d^{r_{k}+1}}{d t^{r_{k}+1}} \tilde{Q}^{(k-1)}(t)\right|_{t=t_{m}} \sim$ $\mathcal{O}(1)$. Therefore, $r_{1, m}^{[k-1]} \sim \mathcal{O}\left(h^{s_{k}+1}\right)$ from (5.13), and $r_{2, m}^{[k]} \sim \mathcal{O}\left(h^{s_{k}+1}\right)$ from (5.14) and Proposition 5.10. Thus, $e_{m+1}^{[k]} \sim \mathcal{O}\left(h^{s_{k}+1}\right)$, completing the inductive proof.

As before, we will prove the smoothness of the rescaled error vector using an inductive argument based on $s$, the degree of smoothness of $\tilde{\tilde{e}}^{k]}$. First, the rescaled error vector has at least 0 degrees of smoothness since $\left\|\tilde{e}^{k]}\right\|_{\infty} \sim \mathcal{O}(h)<\infty$. Assume that $\overrightarrow{\tilde{e}}^{[k]}$ has $s<M+2-s_{k}$ degrees of smoothness in the discrete sense. We will prove that ${\overrightarrow{d_{1}}}^{[k]}$ has $s$ degrees of smoothness, from which we can then conclude that $\overrightarrow{\tilde{e}}^{k]}$ has $(s+1)$ degrees of smoothness. Using (5.11), the divided difference approximation to the derivative of the rescaled error vector satisfies

$$
\left(d_{1} \tilde{e}^{[k]}\right)_{m}=\frac{\tilde{u}_{m}^{[k]}}{h}+\frac{1}{h} \tilde{r}_{1, m}^{[k-1]}-\frac{1}{h} \tilde{r}_{2, m}^{[k]}+\mathcal{O}\left(h^{M+2-s_{k}}\right),
$$

where

$$
\begin{gathered}
\frac{\tilde{u}_{m}^{[k]}}{h}=\sum_{i=1}^{r_{k}} \frac{h^{i-1}}{i !} \frac{d^{i-1}}{d t^{i-1}} \sum_{j=1}^{S-2} \frac{(-1)^{j+1} h^{s_{k}(j-1)}}{j !} \frac{\partial^{j} f}{\partial y^{j}}\left(t_{m}, y\left(t_{m}\right)\left(\tilde{e}_{m}^{[k]}\right)^{j}\right. \\
+\mathcal{O}\left(h^{\left(s_{k}+1\right)(S-2)-1}\right), \\
\frac{\tilde{r}_{1, m}^{[k-1]}}{h}=\sum_{i=0}^{S-r_{k}-1} \frac{h^{i}}{\left(r_{k}+i+1\right) !} \frac{d^{i}}{d t^{i}}\left(\frac{d^{r_{k}}}{d t^{r_{k}}} \tilde{G}\left(t_{m}, \tilde{Q}_{m}^{[k-1]}\right)\right), \\
\frac{\tilde{r}_{2, m}^{[k]}}{h}=\sum_{i=0}^{M+2-s_{k}} h^{i} \sum_{q_{\tilde{Q}}^{i}+q_{t}^{i} \leq i+r_{k}} \zeta_{q_{\tilde{Q}}^{1} q_{t}^{1} \ldots q_{\tilde{Q}}^{n_{i}} q_{t}^{n_{i}}}^{w_{1} w_{2} \cdots w_{n_{i}} w_{f}} \prod_{i=1}^{n_{i}}\left(\tilde{G}_{\tilde{Q}^{q^{i} \tilde{Q} t^{q_{t}^{i}}}}\left(t, \tilde{\Omega}_{m}^{[k]}\right)\right)^{w_{i}} \tilde{G}^{w_{f}}\left(t, \tilde{\Omega}_{m}^{[k]}\right)
\end{gathered}
$$

are computed from (5.12), (5.13), and (5.14).

Using a similar argument from before, $\frac{1}{h} \overrightarrow{\tilde{u}}^{[k]}$ has $s$ degrees of smoothness in the discrete sense. Similarly, $\frac{1}{h} \tilde{\vec{r}}_{1}^{[k]}$ has $\left(M-s_{k}\right)$ degrees of smoothness since $\left.\frac{d^{r_{k}}}{d t^{r_{k}}} \tilde{G}\left(t, \tilde{Q}^{(k-1)}\right)\right|_{t=t_{m}}=\left.\frac{d^{r_{k}+1}}{d t^{r_{k}+1}} \tilde{Q}^{(k-1)}(t)\right|_{t=t_{m}}$ has $\left(M-s_{k}\right)$ degrees of smoothness. Since $\frac{1}{h} \overrightarrow{\tilde{r}}_{2}^{[k]}$ also has $\min \left(M-s_{k}, s\right)$ degrees of smoothness in the discrete sense, we can conclude that $\overrightarrow{d_{1} \tilde{e}^{[k]}}$ has $s$ degrees of smoothness in the discrete sense, which 
implies that $\overrightarrow{\tilde{e}}^{[k]}$ has $\left(M+1-s_{k}\right)$ degrees of smoothness in the discrete sense, completing the inductive proof.

Theorem 5.13 (deferred correction). Suppose $y(t)$, the solution to IVP (2.1), has $S \geq M+1$ degrees of smoothness. Consider a deferred correction method constructed with $(M+1)$ uniformly distributed nodes, $t_{m}=m h, m=0, \ldots, M$, an $r_{0}^{\text {th }}$ order $R K$ method in the prediction step, and $\left(r_{1}, r_{2}, \ldots, r_{k_{l}}\right)^{\text {th }}$ order $R K$ methods for $k_{l}$ correction loops. The local error for the above deferred correction method is $\mathcal{O}\left(h^{s_{k_{l}}+1}\right)$, where $s_{k_{l}}=\sum_{j=0}^{k_{l}} r_{j} \leq M+1$.

Proof. The proof of this theorem is similar to that of Theorem 5.3. The difference involves using the differential form of the error equation (2.6), instead of the Picard integral equation in the correction steps. This leads to a difference in the maximum achievable order. Specifically, deferred correction methods approximate $\frac{\partial}{\partial t} \eta^{(k-1)}(t)$, see (2.6), with at most $M^{\text {th }}$ order accuracy. Thus a deferred correction method can achieve $M^{\text {th }}$ order accuracy at best, unlike the IDC methods, which can achieve $(M+1)^{s t}$ order accuracy.

\section{NumERICAL EXAMPLES}

We test the order of accuracy for various IDC methods constructed using a variety of integrators in the prediction and correction steps. Our numerical runs are in agreement with the analysis in Sections 4 and 5. Then, we compare the stability and accuracy of these methods. Superior stability and accuracy properties are observed for IDC8-RK4 versus IDC8-RK2 and IDC8-FE.

Example 6.1. Consider the IVP,

$$
y^{\prime}(t)=-2 \pi \sin 2 \pi t-2(y-\cos 2 \pi t), \quad y(0)=1
$$

which has an exact solution, $y(t)=\cos 2 \pi t$. We solve this IVP numerically using IDC methods constructed with eight uniformly distributed nodes and various integrators. Specifically, given a final integration time, $T$, and a number of intervals $N$, the IDC method is iterated completely in each interval, $\left[t_{i-1}, t_{i}\right]=T / N, i=$ $1, \ldots, N$ using the quadrature nodes $t_{i, j}=t_{i}+j h, h=H / 7, j=0, \ldots, 7$. This defines the starting value for the next interval. In Table 1, IDC8-FE denotes the IDC method constructed using eight uniformly distributed nodes and forward Euler integrators for the prediction and correction loops, IDC8-RK2 denotes the IDC method constructed using eight uniformly distributed nodes and RK2 integrators for the prediction and correction loops, etc. Eighth order convergence is observed for all IDC8 schemes tested, in agreement with the analysis presented in Sections 4 and 5. Interestingly, the error of the numerical solution obtained using IDC8-RK4 is consistently one order of magnitude smaller than that of those obtained using IDC8-FE and IDC8-RK2. More analysis and discussion on this observation are provided in 3 . The results are summarized in Table 1.

In Table 2, the computations are repeated with deferred correction methods. DC8-FE denotes a deferred correction method constructed using eight uniformly distributed nodes and forward Euler integrators for the prediction and correction loops, DC8-RK2 denotes a deferred correction method with eight uniformly distributed nodes and RK2 integrators for the prediction and correction loops, etc. The expected seventh order convergence is observed for DC8-FE. The eighth order convergence observed for DC8-RK2 and DC8-RK4 is puzzling. This behavior 
does not appear if an odd number of quadrature nodes is used to construct the DC method.

TABLE 1. The error at $T=20$ and the corresponding order of accuracy for various IDC methods used to solve IVP (6.1). Note that the number of steps, $N$, is inversely proportional to the interval size, $H=T / N$, and the subinterval size is $H / 7$.

\begin{tabular}{|c|cc|cc|cc|}
\hline- & \multicolumn{2}{|c|}{ IDC8-FE } & \multicolumn{2}{|c|}{ IDC8-RK2 } & \multicolumn{2}{c|}{ IDC8-RK4 } \\
\hline steps $(N)$ & error & order & error & order & error & order \\
\hline 40 & $5.47 \mathrm{E}-6$ & - & $5.48 \mathrm{E}-6$ & - & $4.49 \mathrm{E}-7$ & - \\
\hline 80 & $1.49 \mathrm{E}-8$ & 8.52 & $1.49 \mathrm{E}-8$ & 8.52 & $1.17 \mathrm{E}-9$ & 8.57 \\
\hline 120 & $5.42 \mathrm{E}-10$ & 8.17 & $5.43 \mathrm{E}-10$ & 8.16 & $4.27 \mathrm{E}-11$ & 8.18 \\
\hline 160 & $5.30 \mathrm{E}-11$ & 8.08 & $5.31 \mathrm{E}-11$ & 8.08 & $4.16 \mathrm{E}-12$ & 8.10 \\
\hline 200 & $8.79 \mathrm{E}-12$ & 8.05 & $8.80 \mathrm{E}-12$ & 8.05 & $6.83 \mathrm{E}-13$ & 8.10 \\
\hline
\end{tabular}

TABLE 2. The error at $T=20$ and the corresponding order of accuracy for various DC methods used to solve IVP (6.1). Note that the number of steps, $N$, is inversely proportional to the interval size, $H=T / N$, and the subinterval size is $H / 7$.

\begin{tabular}{|c|cc|cc|cc|}
\hline- & \multicolumn{2}{|c|}{ DC8-FE } & \multicolumn{2}{c|}{ DC8-RK2 } & \multicolumn{2}{c|}{ DC8-RK4 } \\
\hline steps $(N)$ & error & order & error & order & error & order \\
\hline 40 & $3.89 \mathrm{E}-5$ & - & $5.72 \mathrm{E}-6$ & - & $5.87 \mathrm{E}-7$ & - \\
\hline 80 & $3.30 \mathrm{E}-7$ & 6.88 & $2.60 \mathrm{E}-8$ & 7.79 & $2.54 \mathrm{E}-9$ & 7.85 \\
\hline 120 & $2.15 \mathrm{E}-8$ & 6.74 & $1.02 \mathrm{E}-9$ & 8.00 & $9.83 \mathrm{E}-11$ & 8.02 \\
\hline 160 & $2.91 \mathrm{E}-9$ & 6.94 & $1.02 \mathrm{E}-10$ & 8.00 & $9.81 \mathrm{E}-12$ & 8.01 \\
\hline 200 & $6.11 \mathrm{E}-10$ & 6.99 & $1.70 \mathrm{E}-11$ & 8.00 & $1.64 \mathrm{E}-12$ & 8.01 \\
\hline
\end{tabular}

Definition 6.2. The amplification factor for a numerical method, $A m(\lambda)$, can be interpreted as the numerical solution to

$$
y^{\prime}(t)=\lambda y(t), \quad y(0)=1,
$$

after one time step of size 1 for $\lambda \in \mathbb{C}$, i.e., $\operatorname{Am}(\lambda)=y(1)$.

Definition 6.3. The stability region, $S$, for a numerical method, is the subset of the complex plane $\mathbb{C}$ consisting of all $\lambda$ such that $A m(\lambda) \leq 1$,

$$
S=\{\lambda: A m(\lambda) \leq 1\} .
$$

In Figure 1(a), the stability regions for IDC8-FE, IDC8-RK2 and IDC8-RK4 are computed numerically and plotted. Interestingly, the area of the stability regions increases with the order of the embedded integrator. This isn't overly surprising since the regions of absolute stability for RK methods of order one (Euler's method) through order four increases with the order. A similar observation is made in Figure 1(b) for twelfth order IDC methods constructed using twelve interior points and various integrators. 


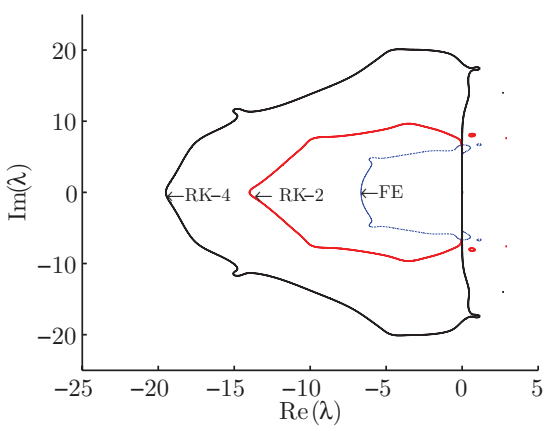

(a) Eighth order IDC Methods

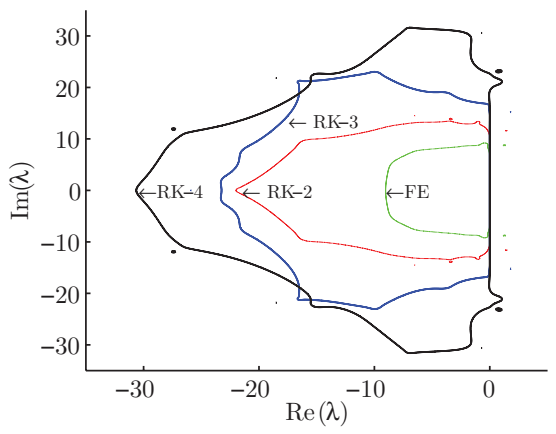

(b) Twelfth order IDC Methods

FiguRE 1. (a) Stability regions for eighth order IDC methods constructed using eight uniformly distributed points and various integrators. The regions of absolute stability increase with the order of the embedded integrator. (b) A similar observation is shown for twelfth order IDC methods.

Definition 6.4. Let $e(\lambda)$ be the error at $T=1$, obtained using a numerical method to solve IVP (6.2), $\lambda \in \mathbb{C}$, with a fixed number of function evaluations (i.e., $d t$ is chosen so that the total number of function evaluations for the method can be controlled). Then, the accuracy plot for that numerical method is defined to be a contour plot of the error, $e(\lambda)$.

Generating the accuracy plots for different methods, where the same number of function evaluations is used to generate each plot, gives us a way to qualitatively, and quantitatively, compare the performance of the different schemes; i.e., for the same amount of work, how accurate are the methods?

In Figures 2(a) and 2(b), the accuracy plots for a generic RK4 method are shown generated for width 56 and 560 function evaluations, respectively. In Figures 2(c) and 2(d), accuracy plots for IDC8-FE are generated with 57 and 570 function evaluations, while Figures 2(e) and 2(f) show the accuracy plots for IDC8-RK4 generated with 57 and 570 function evaluations. For the same number of function evaluations, the accuracy plots show that IDC8-RK4 performs better than RK4 and IDC8-FE. This is particularly evident when $|\lambda| \ll 1$.

\section{Conclusions}

In this paper, a local error analysis is given for SDC methods constructed using general high order RK methods and a uniform distribution of quadrature nodes. Similar arguments apply for the deferred correction method. Numerical examples are in agreement with our analysis.

Our analysis does not extend for non-uniform distributions of quadrature nodes; in fact, preliminary numerical experiments show that the accuracy order of an SDC method, constructed with a non-uniform distribution of quadrature nodes, doesn't always increase with $r$ orders, even when an $r^{t h}$ order RK method is applied. Investigations on using non-uniform quadrature nodes and other high order methods, e.g., multi-step methods, are on-going research topics. 


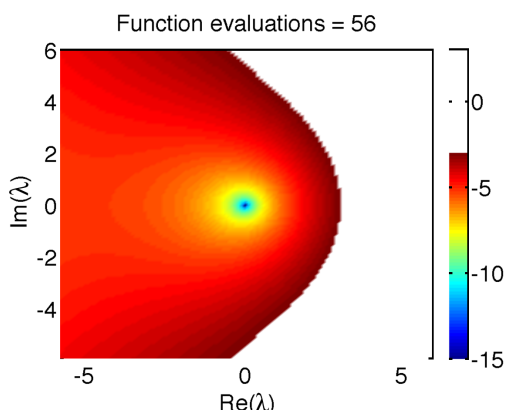

(a) RK4

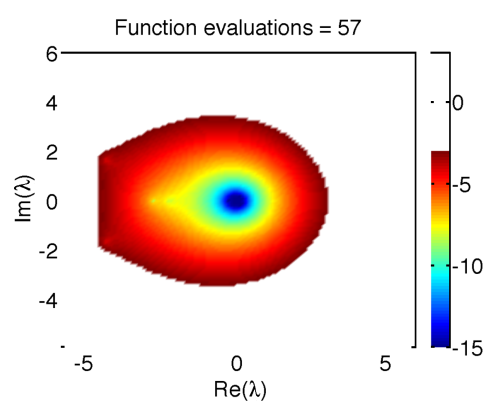

(c) IDC8-FE

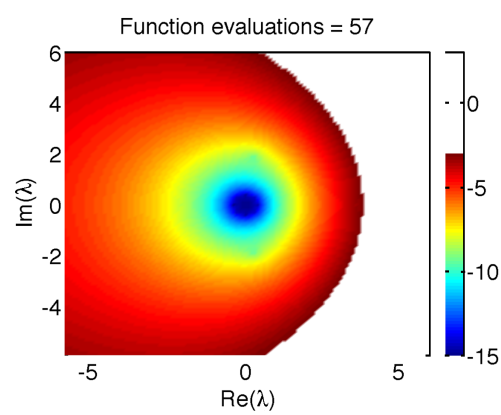

(e) IDC8-RK4

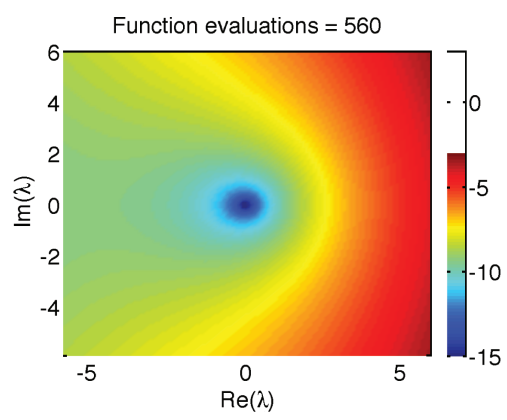

(b) RK4

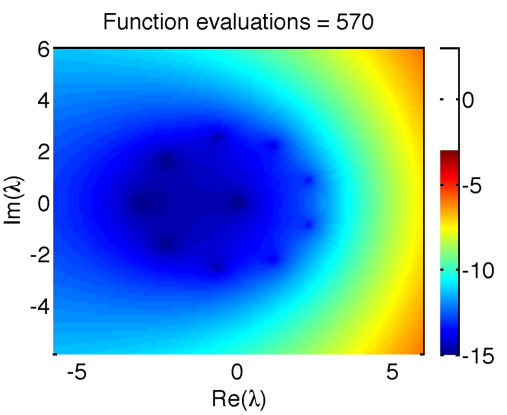

(d) IDC8-FE

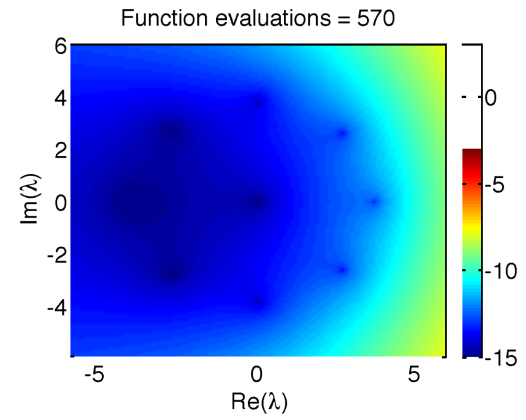

(f) IDC8-RK4

FIGURE 2. The accuracy plots for RK4, IDC8-FE and IDC8-RK4 are generated for $\sim 56$ function evaluations and $\sim 560$ function evaluations.

\section{REFERENCES}

1. W. Auzinger, H. Hofstätter, W. Kreuzer, and E. Weinmüller, Modified defect correction algorithms for ODEs, part I: General theory, Numer. Algorithms 36 (2004), 135-155. MR 2062870 (2005h:65096)

2. Anne Bourlioux, Anita T. Layton, and Michael L. Minion, High-order multi-implicit spectral deferred correction methods for problems of reactive flow, J. Comput. Phys. 189 (2003), no. 2, 651-675. MR.1996061 (2004f:76084)

3. Andrew Christlieb, Benjamin Ong, and Jing-Mei Qiu, A comment on high order integrators embedded within integral deferred correction methods, in preparation. 
4. Alok Dutt, Leslie Greengard, and Vladimir Rokhlin, Spectral deferred correction methods for ordinary differential equations, BIT 40 (2000), no. 2, 241-266. MR1765736 (2001e:65104)

5. Thomas Hagstrom and Ruhai Zhou, On the spectral deferred correction of splitting methods for initial value problems, Commun. Appl. Math. Comput. Sci. 1 (2006), 169-205 (electronic). MR 2299441

6. A. Hansen and J. Strain, Convergence Theory for Spectral Deferred Correction, preprint, University of California at Berkeley, February (2005).

7. A. C. Hansen and J. Strain, On the order of deferred correction, http://www.damtp.cam. ac.uk/user/na/people/Anders/Deferred.pdf.

8. Jingfang Huang, Jun Jia, and Michael Minion, Accelerating the convergence of spectral deferred correction methods, J. Comput. Phys. 214 (2006), no. 2, 633-656. MR 2216607 (2006k:65173)

9. - Arbitrary order Krylov deferred correction methods for differential algebraic equations, J. Comput. Phys. 221 (2007), no. 2, 739-760. MR2293148 (2008a:65134)

10. Anita T. Layton and Michael L. Minion, Conservative multi-implicit spectral deferred correction methods for reacting gas dynamics, J. Comput. Phys. 194 (2004), no. 2, 697-715. MR 2034861 (2004k:76089)

11. Implications of the choice of quadrature nodes for Picard integral deferred corrections methods for ordinary differential equations, BIT 45 (2005), no. 2, 341-373. MR2176198 (2006h:65087)

12. A.T. Layton, On the choice of correctors for semi-implicit Picard deferred correction methods, Applied Numerical Mathematics 58 (2008), no. 6, 845-858. MR2420621

13. A.T. Layton and M.L. Minion, Implications of the choice of predictors for semi-implicit Picard integral deferred corrections methods, Comm. Appl. Math. Comput. Sci. 1 (2007), no. 2, 1-34. MR 2327081 (2008e:65252)

14. Yuan Liu and Chi-Wang Shu, Strong stability preserving property of the deferred correction time discretization, J. Comput. Math. 26 (2008), no. 5, 633-656. MR2444722

15. Michael L. Minion, Semi-implicit spectral deferred correction methods for ordinary differential equations, Commun. Math. Sci. 1 (2003), no. 3, 471-500. MR2069941 (2005f:65085)

16. Semi-implicit projection methods for incompressible flow based on spectral deferred corrections, Appl. Numer. Math. 48 (2004), no. 3-4, 369-387, Workshop on Innovative Time Integrators for PDEs. MR 2056924

17. Robert D. Skeel, A theoretical framework for proving accuracy results for deferred corrections, SIAM J. Numer. Anal. 19 (1982), no. 1, 171-196. MR646602 (83d:65184)

18. Yinhua Xia, Yan Xu, and Chi-Wang Shu, Efficient time discretization for local discontinuous Galerkin methods, Discrete Contin. Dyn. Syst. Ser. B 8 (2007), no. 3, 677-693 (electronic). MR2328730

Department of Mathematics, Michigan State University, East Lansing, Michigan 48824

Department of Mathematics, Michigan State University, East Lansing, Michigan 48824

Department of Mathematics and Computer Sciences, Colorado School of Mines, Golden, Colorado 80401

E-mail address: jingqiu@mines.edu 